\title{
Detection of Spatio-Temporal Evolutions on Multi-annual Satellite Image Time Series: A Clustering Based Approach
}

\author{
Lynda Khiali ${ }^{\mathrm{a}}$, Mamoudou Ndiath ${ }^{\mathrm{a}}$, Samuel Alleaume ${ }^{\mathrm{a}}$, Dino Ienco ${ }^{\mathrm{a}, \mathrm{b}}, \mathrm{Kenji}_{\mathrm{Ose}}^{\mathrm{a}}$, Maguelonne Teisseire $^{\mathrm{a}}$ \\ ${ }^{a}$ TETIS, APT, CIRAD, CNRS, IRSTEA, UNIV.Montpellier, Montpellier, France \\ ${ }^{b}$ LIRMM, Montpellier, France
}

\begin{abstract}
The expansion of satellite technologies makes remote sensing data abundantly available. While the access to such data is no longer an issue, the analysis of this kind of data is still challenging and time consuming. In this paper, we present an object-oriented methodology designed to handle multi-annual Satellite Image Time Series (SITS). This method has the objective to automatically analyse a SITS to depict and characterize the dynamic of the areas (the way that the land cover of the areas evolve over time). First, it identifies the spatio-temporal entities (reference objects) to be tracked. Second, the evolution of such entities is described by means of a graph structure and finally it groups together spatio-temporal entities that evolve similarly. The analysis were performed on three study areas to highlight inter (among the study areas) and intra (inside a study area) similarity by following the evolution of the underlying phenomena. The analysis demonstrate the benefits of our methodology. Moreover, we also stress how an expert can exploit the extracted knowledge to pinpoint relevant landscape evolutions in the multi-annual time series and how to make connections among different study areas.
\end{abstract}

Keywords: Satellite image time series; Object-oriented image analysis; Clustering; Graph Analysis; Inter-site analysis

\section{Introduction}

Nowadays, remotely sensed satellite images constitute a rich source of information that can be leveraged to support several applications including food risk prevention, land use planning and mapping, natural habitat monitoring, land cover classification and many other several tasks [1]. Advances in satellite technologies 5 and the high images acquisition frequency result into huge amount of remote sensing data that need effective and efficient analytics approaches. Standard tools including photo interpretation and fully supervised approaches are not suitable for dealing with this large amount of spatio-temporal data 2. In this context, data mining techniques have already shown their usefulness 3 and they seem adequate to extract valuable knowledge to support remote sensing analysis on both single image or Satellite Image Time Series (SITS) data [4, 5, 6].

Several data mining approaches are commonly employed to analyse SITS data: supervised [7, unsupervised [8, 9] and semi-supervised methods [10, 11]. Due to the lack of reference data, unsupervised methods such as clustering are widely employed to explore and mine patterns from remote sensing data. Data clustering is the process of partitioning the data into groups where, similar examples are assigned to the same group and non similar examples are assigned to different ones 12 .

Email addresses: lynda.khiali@irstea.fr (Lynda Khiali), ndiathmamoudou@yahoo.fr (Mamoudou Ndiath), samuel.alleaume@irstea.fr (Samuel Alleaume), dino.ienco@irstea.fr (Dino Ienco), kenji.ose@irstea.fr (Kenji Ose), maguelonne.teisseire@irstea.fr (Maguelonne Teisseire) 
In this work, we propose an unsupervised method to analyse SITS data at object level in order to highlight intra and inter similarity among the different study areas. Two important factors influence the clustering of remote sensing time series: i) the distance measure choice and ii) how data are represented.

Considering distance/similarity measures commonly involved in SITS analysis, the standard choice is the euclidean distance (or one of its variants) 13 due the fact that it is intuitive, easy to implement and parameter free. Conversely, how to represent SITS data in order to exploit as much as possible the available spatio-temporal information remains a challenge and researchers have devoted time and effort to deal with this point [14, 15].

In unsupervised as well as in the supervised analysis, SITS data are mainly exploited at pixel level. For each pixel, a time series is built. The radiometric values of the pixel, in the considered satellite images, are gathered and ordered based on the temporal dimension. Thus, the number of time series is equal to the number of pixels covered by the considered area [14. The method proposed in [6] analyzes SITS information at pixel level to detect land cover classes. To perform clustering, they compute a distance matrix between pixel time series using the euclidean distance. Then, they run the Affinity Propagation (AP) algorithm [16]

30 on the matrix. The method was evaluated on three data sets, the AP results were compared to those obtained using the K-means and the Agglomerative Hierarchical clustering algorithms. The authors introduce in 17 a method to analyse multi-annual satellite image time series to monitor vegetation areas using the vegetation index. The method analyses the SITS at pixel level, it builds an annual profile which characterizes the pixel evolution on each year. Then, it gathers these representative annual profiles to construct a final sequence to

35 characterize the evolution of a pixel over several years. Finally, a clustering algorithm based on the euclidean distance is employed to characterize the vegetation dynamic.

Unlike previous methods, [5] introduces a pixel-based time series clustering exploiting Dynamic Time Warping (DTW) [18. The authors used DTW combined with K-means to deal with unaligned time series data that are also characterized by missing values due to cloud phenomena.

Unlike the method presented above, object-based representation can be exploited to analyse satellite images [19]. Objects stand for meaningful structures in the image. They are identified using a segmentation algorithm 20 that spatially groups similar pixels, based on homogeneity criteria.

In the literature, object-oriented representation is rarely employed to analyse SITS data [15] while it is widely used for single image analysis 21. In fact, aligning pixels between two consecutive images (at the 45 same resolution) is straightforward as you only have to superpose the images on the pixel grid. Conversely, aligning objects coming from different images, from the same time series, can be difficult due to the landscape evolution.

A first step in this direction is presented in [14 where both pixel and object representations are exploited to perform an unsupervised land cover characterization. This method enriches the pixel time series using 50 the corresponding object information. The idea is to include spatial context as information for each pixel. The analysis show that enriching pixels with contextual features improve the final results.

Most of the proposed methods in the literature perform object-oriented analysis on a time series by comparing two images from two successive timestamps. The method proposed in 21 introduces an objectoriented approach combining two satellite images of the same area acquired by different sensors. The spectral bands of the satellite images are first stacked, then the resulting image is segmented. The goal is to monitor land cover changes, thus the segmentation result is classified into land cover change classes to produce a change map.

The method proposed in 22 performs segmentation on a multidate image where the whole set of spectral bands of the time series is stacked together. This approach integrates the spatial, spectral and temporal dimensions of the data to identify coherent objects. The method is especially tailored to separate forest changes between areas that changes and areas that do not. An object is validated as changed if more than a fixed threshold of its area is covered by a forest change. Similarly to the previous method, also in this case the result is a change map highlighting the area where the forest cover changed.

Recently, 15] proposes an object-oriented approach that tracks spatio-temporal phenomena and extracts evolution patterns. This method aims to automatically detect and extract spatio-temporal information from SITS data. It segments each satellite image independently, then it links together the resulting objects between two consecutive timestamps of the time series. The method results on a set of temporal profiles 
representing the evolution of the object attributes. An evolution map is then produced, such map can be used to detect the most and the less stable areas within a study site.

In [23, the authors have proposed an object-oriented clustering method that leverage the object-oriented time series representation proposed in [15], Our approach uses the representation introduced in [15] to embed the spatio-temporal entities detected in the SITS data. Then, similar spatio-temporal evolutions are grouped together using a clustering algorithm. The experiments were conducted on two study sites, independently, considering small time series (six images) covering a period of eight months.

In this paper, conversely to the work proposed in [23], we deal with the challenging analysis of multiannual SITS data with the purpose to extract inter (among the study areas) as well as intra (inside a study area) similarity among spatio-temporal phenomena. To this end: i) we adapt the strategy proposed in [15] to extract evolution patterns from multi-annual SITS, ii) we design a novel distance measure between two spatio-temporal phenomena and iii) we define a framework that make connections among different study areas allowing inter-site analysis.

Unlike common time series analysis methods that mainly focus their effort to discriminate between changed and unchanged area, we propose an unsupervised approach to group together spatio-temporal phenomena that evolve similarly in the multi-annual time series and among different study areas.

Experiments are carried out on multi-annual SITS data (Spot-2, Spot-4 and Spot-5) describing three different study areas. In our analysis, we demonstrate the quality of the proposed framework to accomplish inter and intra-site analysis. While in the former we focus our attention on phenomena that evolve similarly inside a particular site; in the latter we point out cross-site similarity among the different study areas. The cross-site analysis shows that spatio-temporal phenomena belonging to different study areas can be grouped together establishing some kind of connections among areas. This kind of analysis can be particularly useful in the case an expert starts to explore and inspect an unknown study site leveraging previous acquired knowledge on a spatially closed (well-known) area.

This article is organized as follows. Section 2 describes the three study areas and the preprocessing we adopt on the data. The proposed method is introduced in Section 3 . Section 4 reports and discusses the obtained results. The conclusion of our study is drawn in Section 5 .

\section{Data}

\subsection{Study area}

Three study site located in the south of France were involved in our study: (A) Lower Aude Valley, (B) Mountain of the Moure and Causses of Aumelas and (C) Pic Saint Loup. Figure 1 shows the geographical position of these three sites.

A. Lower Aude Valley

The Lower Aude valley (Fig 2(a)] is a Natura 2000 site located in the terminal section of the Aude River. Before reaching the Mediterranean Sea, the Aude River runs through a flat wetland area of about 4842 ha. From a biodiversity point of view, $56 \%$ of the site is composed of Natural Habitat types of Community Interest (NHCI). In total, 19 NHCI are located on the site, including 5 priority habitat types. The most widespread habitats are Mediterranean saltmarshes and Saline coastal lagoons. The remaining area (43.7\%) is principally composed of vineyards, cereal crops and temporary or permanent meadows. One more characteristic of the site is its exposure to flooding events (mostly during winter) as well as to drought episodes (maximum intensity occurring in the end of summer). The floodable areas are situated predominantly around the two coastal lagoons: Vendres in the north part of the site and Pisse-Vaches in the south. The Mediterranean sea also influences the salinity across the site (soils and water bodies), with a general gradient increasing from northwest to southeast.

B. Mountain of the Moure and Causses of Aumelas

The Mountain of the Moure and Causses of Aumelas (MMCA) (Fig 2(b) is located at the west of Montpellier (France). Considered as a Natura 2000 site, it has a surface of around 9369 ha and it is 


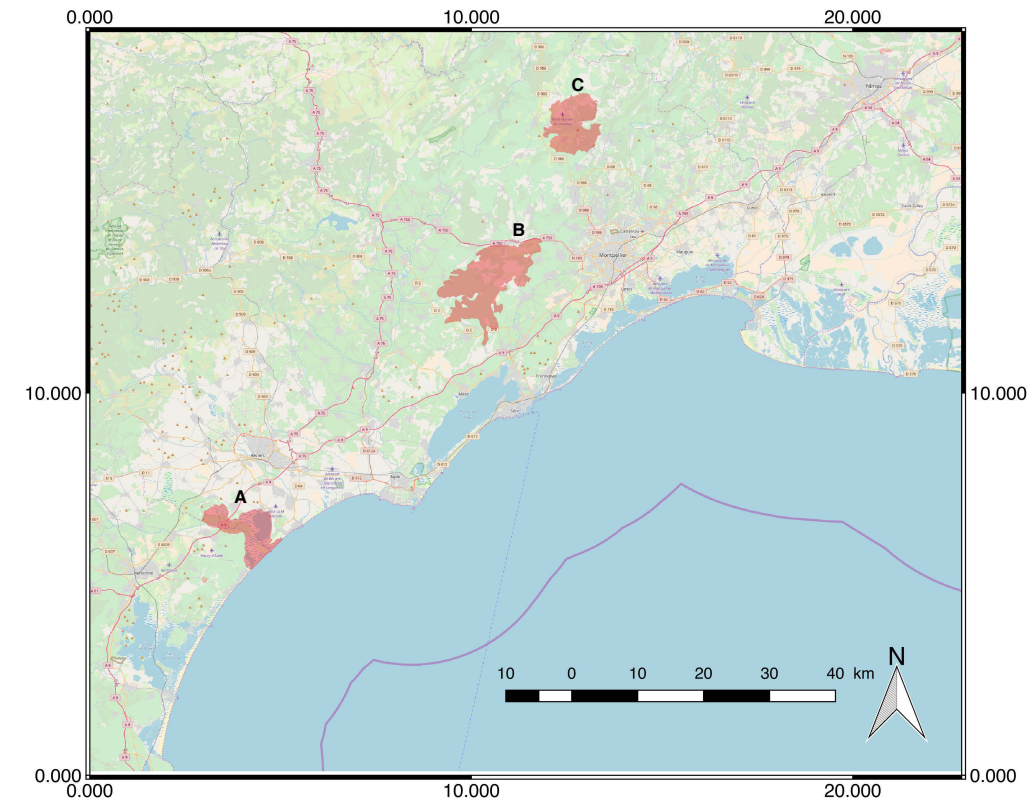

Figure 1: Location of the selected study sites ((A) Lower Aude Valley; (B) Mountain of the Moure and Causses of Aumelas; (C) Pic Saint Loup Natura 2000 site).

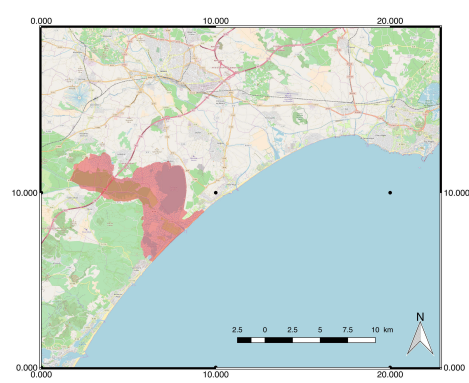

(a)

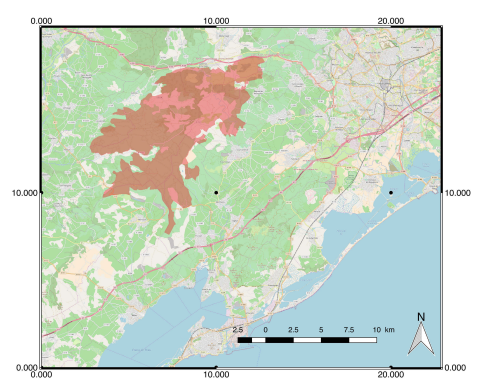

(b)

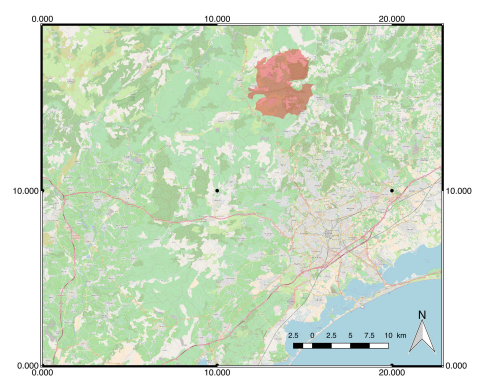

(c)

Figure 2: Location of the (a) Lower Aude Valley, (b)Mountain of the Moure and Causses of Aumelas and (c) Pic Saint Loup.

situated between 3 basins: the agglomeration of Montpellier, the Thau basin and the Herault valley. Agricultural activities (particularly pastoral activity), fires and rural character of the site make it the largest garrigue territory of the Herault department. This site is characterised by the predominance of natural areas and open habitats. Forests cover its upper part, while the middle is dominated by garrigue vegetation, as for as the south which is characterized by agricultural areas. The MMCA site includes also numerous Mediterranean ponds that are spread over it.

\section{Pic Saint Loup}

Located 20 kilometers north from the city of Montpellier (France), Pic Saint Loup (Fig 2(c)p is a Natura 2000 site. It has a surface around 4420 ha and covers 8 municipalities: Cazevieille, Mas de Londres, Notre Dame de Londres, Rouet, Saint Jean de Cuculles, Saint Martin de Londres, Saint Mathieu de Treviers and Valflaunes. The Pic Saint Loup site presents a mosaic of land cover classes which includes expanses of lawns and matorrals, rock scarps, forest, cultivated plains, etc. The lawns 
are covered by different plants as gagea granatelli/lacaitae. Forest is characterised by green oak, especially in the southern part of the site which is marked by the Mediterranean influences. Note also that human activity such as pastoralism, tree cutting and wood exploitation have greatly contributed to the current landscape.

To perform an inter-site analysis on the three sites described bellow, we exploit satellite images of different sensors: Spot-2, Spot-4 and Spot-5. These satellite images are available in the context of the Spot World Heritage program $(\mathrm{SWH}) 1$. Such images are filtered with the aim to select cloud-free images. We construct an image dataset composed of three satellite image time series : 53 images for the Lower Aude Valley area, 46 images for the MMCA site and 50 images depicting the Pic Saint Loup area. The selected images span over a period of eighteen years, from 1990 to 2008, considering each year the interval between May and September. The dataset description is reported in the table 1 .

\begin{tabular}{|l|l|l|l|l|}
\hline & Spot-2 & Spot-4 & Spot-5 & Total \\
\hline Lower Aude Valley & 29 & 16 & 8 & 53 \\
\hline MMCA & 21 & 17 & 8 & 46 \\
\hline Pic Saint Loup & 25 & 17 & 8 & 50 \\
\hline Total & 75 & 50 & 24 & 149 \\
\hline
\end{tabular}

Table 1: The number of satellite images acquired for each study area per sensor (Spot-2, Spot-4 and Spot-5).

The number of satellite images acquired per year and per month on the Lower Aude Valley, MMCA and the Pic Saint Loup sites is illustrated in Figures 3 , 4 and 5 respectively.

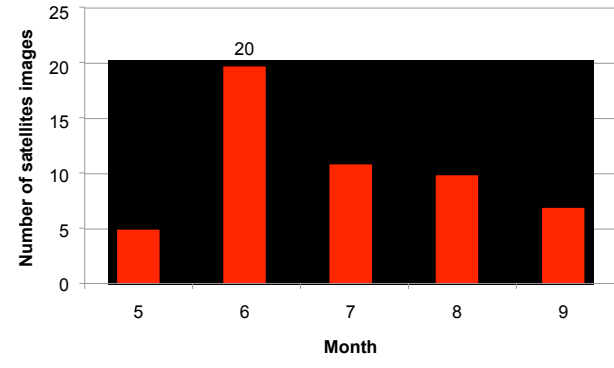

(a)

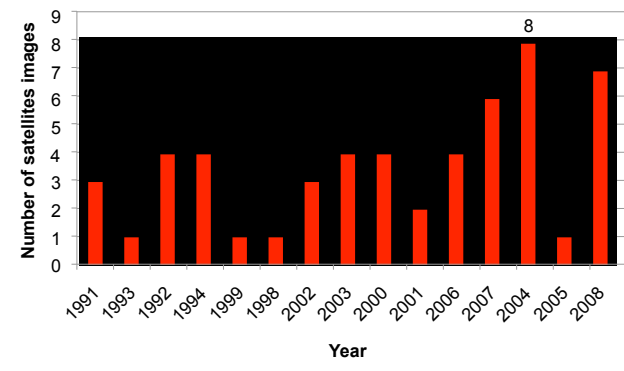

(b)

Figure 3: The number of satellite images acquired per month (a) and per year (b) on the Lower Aude Valley

\subsection{Data Preprocessing}

In the preprocessing step, the images are first filtered. The cloudy images are identified and removed from the dataset, then resampled. The resampling process consists on modifying the image resolution by either increasing (oversampling) or decreasing (subsampling) it. In our case, all the images were resampled to 20 meters using the bilinear interpolation method. The objective is to create an homogeneous satellite image dataset. In fact, we use images of different sensors: Spot-2 and Spot-4 provide images with a resolution of 20 meters, while Spot-5 supplies images with a resolution of 10 meters. Next, radiometric information is extracted from satellite images. Successively, a segmentation is performed on them. Finally, a field expert categorize the resulting segments.

\footnotetext{
${ }^{1}$ The associate satellite images are available for the research community: https://www.theia-land.fr/fr/projets/spot-worldheritage
} 


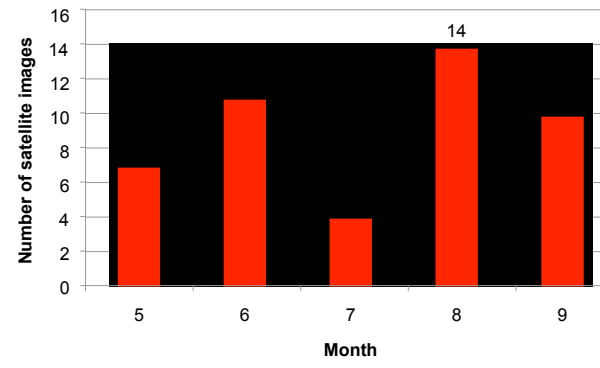

(a)

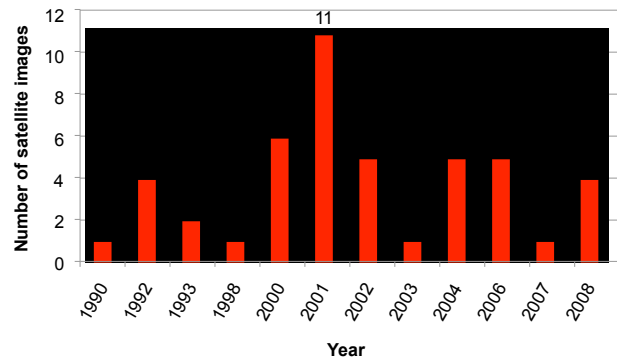

(b)

Figure 4: The number of satellite images acquired per month (a) and per year (b) on the MMCA

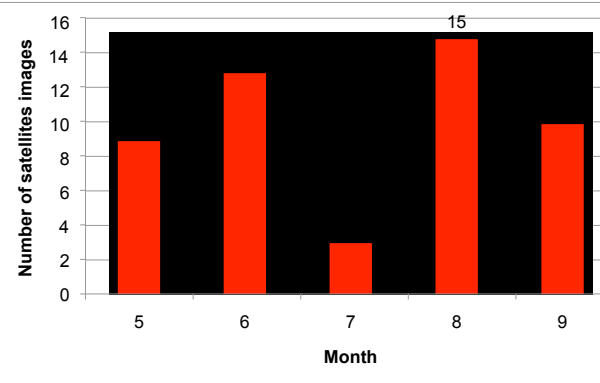

(a)

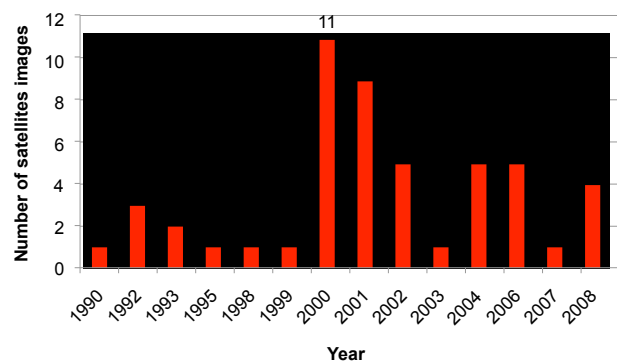

(b)

Figure 5: The number of satellite images acquired per month (a) and per year (b) on the Pic Saint Loup

\subsubsection{Extraction of the Radiometric Information}

In our study, we use two types of radiometric information: (i) the spectral bands and (ii) the spectral indices. To have a compatible dataset, we keep the common spectral bands on the Spot satellite images: Red, Green and Near InfraRed (NIR). These raw bands are enriched with spectral indices. In this work we compute five spectral indices compatible with Spot satellite images.

- The Soil-Adjusted Vegetation Index (SAVI): Involving two spectral bands, red and NIR, the SAVI [24] index is useful to detect soil and vegetation. It allows to normalize soil variation (dark soil, light soil) avoiding to influence the vegetation spectral response.

- The Brightness Index (BI): The importance of this index appears principally in its potential to show the different situations of soils, by representing their different reflectance and brightness. In fact, beaches and parking lots take the highest value while water area and wet area take the lowest values whereas, agriculture plot shows average values.

- The Color index (CI): The CI 25] index characterises the soil, it is computed using the green and red channels. In most cases, CI gives complementary information with the BI and the NDVI indices for a better understanding of the soil surfaces evolution.

- The Normalized Difference Water Index II: The NDWI2 26] uses the green and the NIR bands. It allows to characterise the wet and water areas on remote sensing images.

- The Normalized Difference Vegetation Index: The NDVI 27 aims to characterize the vegetation cover. It ranges in the interval $[-1,1], 1$ stands for vegetation canopy, while 0 stands for bare soil. It is computed using the red and the NIR bands. 
Table 2: The segmentation parameters of the three study areas.

Different segmentation parameters are used for each study area, thus to identify the landscape unit of each site. Our objective is to define compact segments that represent the different entities of the area such us the agriculture plots, forest, etc. The field expert validate the segmentation results by photo interpretation. 


\subsection{Ground Truth Data}

The satellite images are analysed by a field expert to identify their different land cover classes. Observing the three sites (Lower Aude Valley, MMCA and Pic Saint Loup), the expert defines 9 classes. As showed in Figure 6, the three sites have in common 3 classes: Crop, Shrubby vegetation and Vineyard. Therefore, the two sites Pic Saint loup and MMCA have two shared classes: Open space with few or no vegetation and Forest. While the class Artificial area is specific to the Pic Saint Loup site, the land cover classes : Beach, Water area and Wet area only appear in the Lower Aude valley site.

The land cover classes repartition in the three study areas is shown in Figure 7. The Pic Saint Loup and the $M M C A$ are mainly characterized by natural habitats as Forest and Shrubby vegetation while, the Lower Aude Valley by agricultural plots (Crop and Vineyard), wetlands (Water area and Wet area).

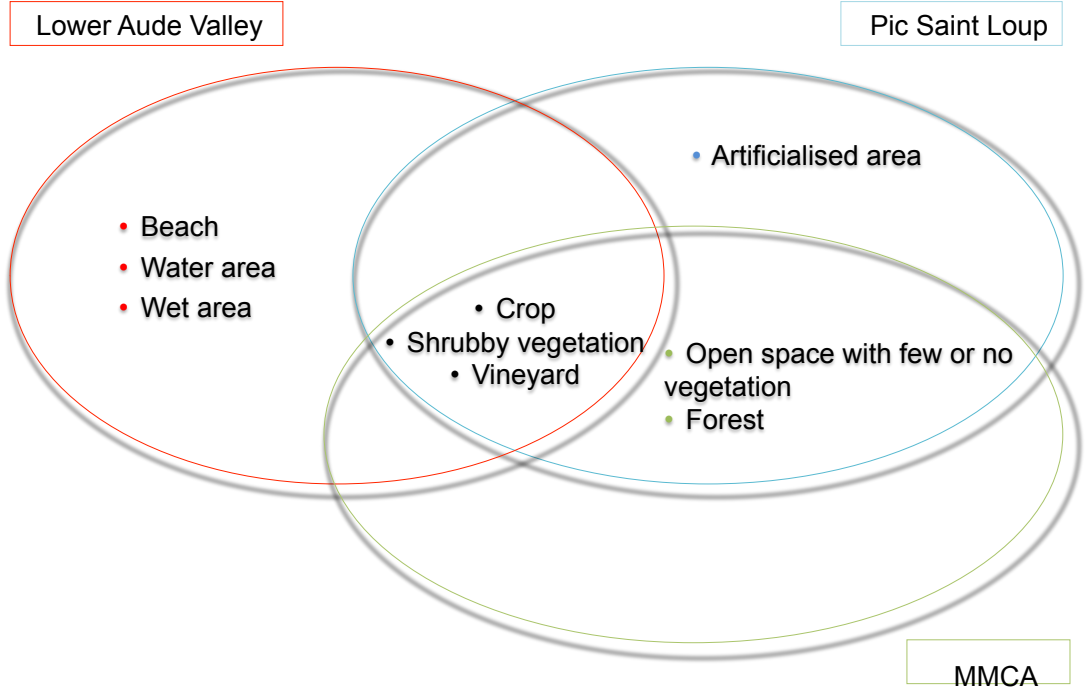

Figure 6: The identified land cover classes in the three sites. The classes in common in the study areas are: Crop, Shrubby vegetation and Vineyard.

\section{Method}

In this section, firstly we supply a general overview of our approach, then we detail the different steps of the proposed framework.

\subsection{Workflow}

Figure 8 visually summarizes the different steps of the methodology. The framework takes as inputs the raw satellite image time series and its segmentation (Fig 8 (Input)). First, the segments are filtered to detect a subset of objects, named reference objects (Fig 8 (Step 1)). The reference objects stand for the pertinent spatio-temporal entities to analyse. Then, for each reference object, an evolution graph (Fig 8 (Step 2)) is built using the segments overlapping it in the SITS. The evolution graph describes the behaviour of the reference object over the time. The graph construction procedure is performed for all the detected reference objects. Finally, a clustering algorithm is applied to detect similar behaviour among evolution graphs (Fig 8 (Step 3)).

The two first steps of our framework are inspired by the method proposed in [15. In their work, the authors deploy the strategy on annual time series. Our goal is the analysis of multi-annual SITS data, to this purpose we extend such approach to fit our context. 


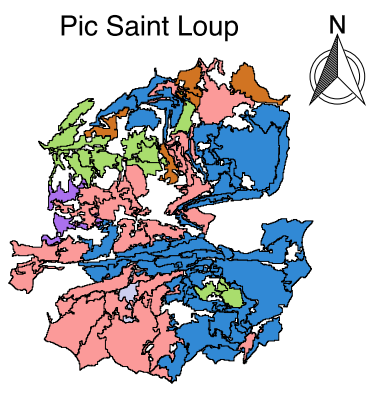

$\begin{array}{lllllll}1 & 0 & 1 & 2 & 3 & 4 & \mathrm{~km}\end{array}$

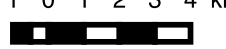

$\square$ Artificialised area
$\square$ Crop
Forest
$\square$ Open space with few or no vegetation
Shrubby vegetation
Vineyard

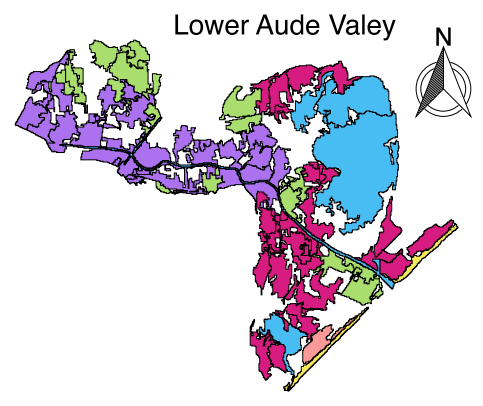

$\begin{array}{lllllll}1 & 0 & 1 & 2 & 3 & 4 & \mathrm{~km}\end{array}$
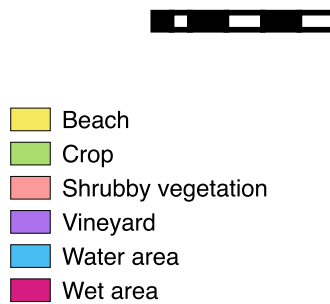
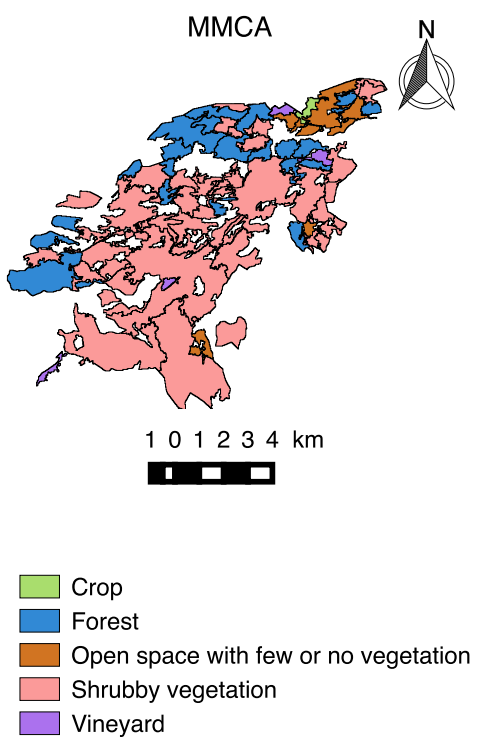

Figure 7: The repartition of the land cover classes in the three sites. The classes in common in the study areas are: Crop, Shrubby vegetation and Vineyard.

\subsection{Preliminaries and Notations}

For each study area, we consider a satellite images time series covering the area. A SITS is defined as an ordered set of satellite images covering the same area, $\left(I_{1}, I_{2}, \ldots, I_{t}, I_{t+1}, \ldots, I_{T}\right)$, where the order depends on the acquisition timestamps $(1,2, \ldots, t, t+1, \ldots, T)$ of the images.

In addition to raw satellite image time series, we consider also their segmentation. We define for each image $I_{t}$ a set of segment/object, $O_{t}=\left\{o_{t}^{i}\right\}_{i=1}^{\left|O_{t}\right|}$, where $i$ denotes the segment identifier. We associate also to each object, $o_{t}^{i}$, a set of pixels it covers, Pix $\left(o_{t}^{i}\right)$, and a vector of its radiometric information, Info $\left(o_{t}^{i}\right)$.

As the images are segmented independently, each image has a different number of segments. We note with $\mathcal{O}$ the union of all the identified segments in the SITS, $\mathcal{O}=\bigcup_{t=1}^{T} O_{t}$.

\subsection{Reference Object Selection}

The first step of the framework is the selection of reference objects [15]. The reference objects are segments standing for phenomena of interest we want to track (spatio-temporal entities). A reference object can be for example an agriculture parcel, in this case the dynamic to track is the growing cycle of the plantations.

At this step, the method analyses the entire set of segments resulting from the satellite images time series segmentation and selects informative objects covering the whole study area.

The intuition behind the reference object selection is the identification of the maximal extent of a certain phenomenon. Thus, we made the assumption that a phenomenon has a dynamic that results on different spatial extent in term of size (the number of pixel covered). This phenomenon reaches its maximal extent at a certain timestamps at least once over the considered period. For instance, if we consider a temporary lake, the lake covers the biggest area over the year in winter. While, during the other seasons (especially in summer) the water will cover a smaller area. Thus, the covered area in winter will be segmented into different objects. The reference object, in this case, is the segment associated to the area covered in winter which corresponds to the maximal extent of the lake. For this reason, the reference object selection step attempts to select the biggest (non overlapping) segments in the satellite image time series. 
Author-produced version of the article published in

International Journal of Applied Earth Observation and Geoinformation, 2019, N 74, p. 103-119

The original publication is available at

http://www.sciencedirect.com/science/article/pii/S0303243418304781

Doi: 10.1016/j.jag.2018.07.014

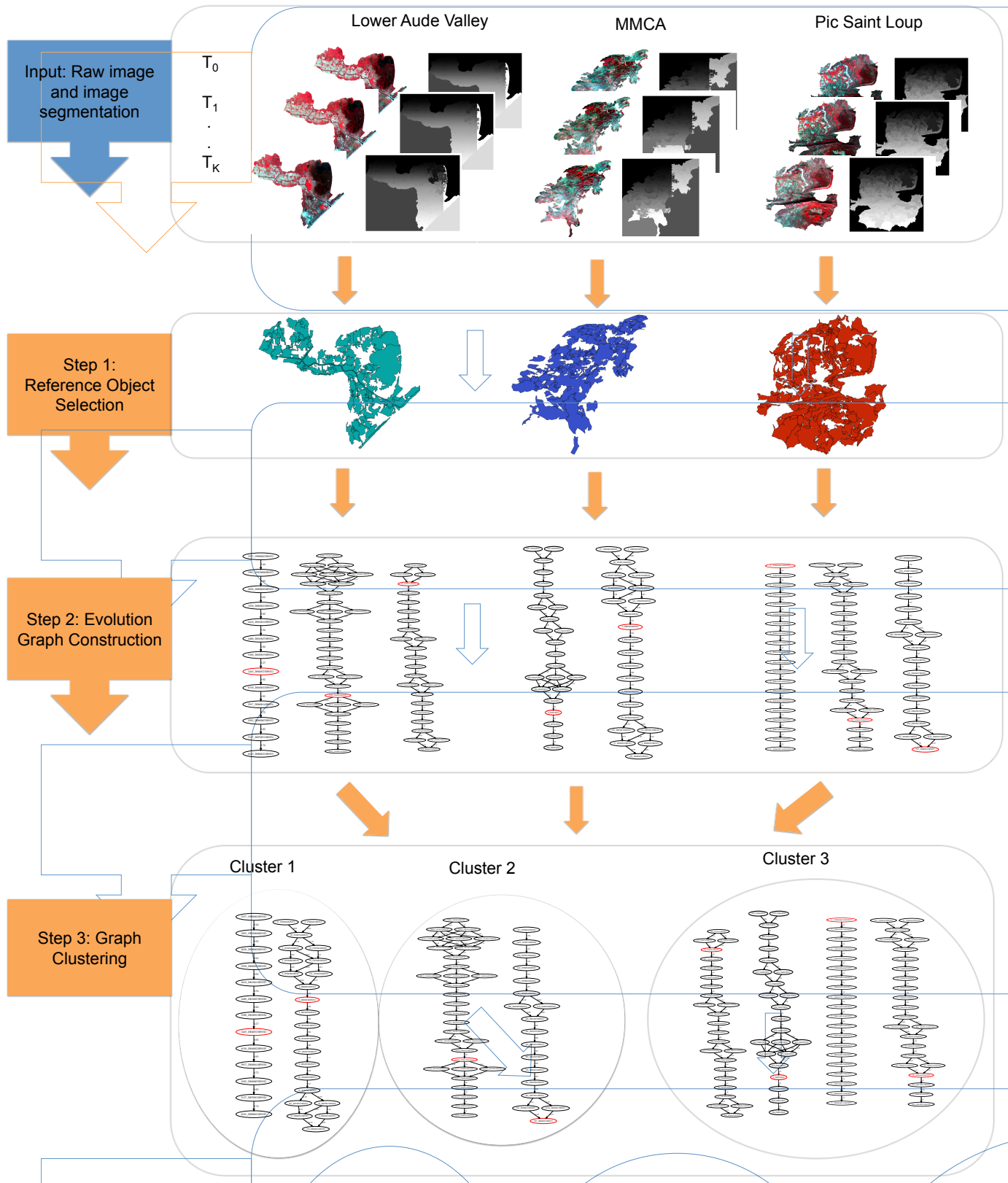

Figure 8: Overview of the proposed framework to detect and cluster the evolution graphs: (Step1) Reference Object Selection, (Step2) Evolution Graph Construction and (Step3) Graph Clustering . 
The reference object selection is treated as a covering problem [15. We select the subset of segments that cover as much as possible the study area while minimizing their overlapping. In fact, the $T$ images composing the time series cover the same area (the same grid of pixel), a pixel is then covered by $T$ segments. Thus, the selected objects may overlap.

More in detail, the method iterates over the set of segments $O$, starting from an empty set of reference objects $R$. At each iteration it selects one object from $O$ and adds it to $R$. Following the previous main assumption, the method selects the biggest object in the time series. To identify these objects, the segments are weighted. The weight is supplied by the following function:

$$
\text { weight }(o)= \begin{cases}|\operatorname{Pix}(o)|, & \text { if } \operatorname{Novelty}(o)=1 \\ \operatorname{Novelty}(o), & \text { if } \alpha<\operatorname{Novelty}(o)<1 \\ 0, & \text { if } \operatorname{Novelty}(o)<\alpha\end{cases}
$$

Where:

- $\operatorname{Pix}(\mathrm{o})$ is the set of pixels covered by the segment o

- Novelty(o) is the rate of pixels covered only by the segment $o$ considering the set of objects $R$.

- $\alpha$ is a threshold that defines the minimum value of Novelty a segment must exhibit to be considered.

\subsection{Graph Construction}

In this step, a graph-based representation, called evolution graph, is employed to describe the dynamic of the selected reference objects. An evolution graph is a directed acyclic graph. For each reference object, $o^{*}$, we construct an evolution graph, named $G\left(o^{*}\right)$, defined by a set of nodes $\left(V_{O^{*}}\right)$ and a set of edges $\left(E_{O^{*}}\right)$, $G_{o^{*}}=\left(V_{o^{*}}, E_{o^{*}}\right)$

The idea is to track the state of a reference object, $o^{*}$, considering the set of satellite composing the time series. Therefore, the spatial extent of the reference object is projected on the satellite images and its overlapping segments are selected i.e. a group of segments covering the same pixels as the reference object is selected on each image. 
In the work proposed in [15, the authors employ the full set of images belonging to the time series in order to derive the corresponding evolution graphs. This was done since the considered time series is short (only six dates) and the goal was to perform phenological analysis. Conversely, in our context we are interested to mine evolution graphs from multi-annual time series. Due to this fact, using all the images of the time series may introduce redundancy, since the difference between the acquisition timestamp of some mages is neglectable (i.e. considering the MMCA site: 21-08-2001, 22-08-2001 and 27-08-2001).

To deal with this fact, in order to highlight useful evolutions in the land cover reducing redundancy in the information described by the evolution graphs, we need to discard too similar images (according to their date of acquisition). According with field expert recommendations, we set up a time gap of two months between two successive images. Starting from the reference object time stamps, we choose satellite images that are far away (considering the date of acquisition) of at least two months from the previous date of the series.

Considering the spatial extent, the segments to consider, in the evolution graph construction, are those having most of their extent included in the extent of the reference object. The objective is to avoid as much as possible the noisy segments and the non-representative ones. Therefore two thresholds are defined, $\sigma_{1}$ and $\sigma_{2}$ :

$$
V_{o^{*}}=\left\{o \mid o \in O, \frac{\left|\operatorname{Pix}\left(o^{*}\right) \cap \operatorname{Pix}(o)\right|}{|\operatorname{Pix}(o)|} \geq \sigma_{1} \text { or } \frac{\left|\operatorname{Pix}\left(o^{*}\right) \cap \operatorname{Pix}(o)\right|}{\left|\operatorname{Pix}\left(o^{*}\right)\right|} \geq \sigma_{2}\right\}
$$

Where:

- $O$ is the set of segment

- $o$ is a segment on $O$

- $o^{*}$ is a reference object

- Pix(o) is the set of pixels covered by the segment o

- Pix $\left(o^{*}\right)$ is the set of pixels covered by the reference object $o^{*}$

The first parameter $\left(\sigma_{1}\right)$ is the most significant and allows the selection of segments that should present most of their spatial extent inside the reference object extent. The second parameter $\left(\sigma_{2}\right)$ is used to retain all the objects covering more than a certain percentage of the reference object extent.

Once the representatives segments of the reference object are selected, they are organized and linked to form an evolution graph. The segments are first grouped considering their images timestamps i.e. each group will contain all the segments belonging to the same image of the time series. Then, the segments groups are sorted in increasing order considering the timestamps (acquisition date) of their image. Finally, the segments of each two successive groups are connected by links. To link the segments we consider always their extent linking overlapping ones. Thus, segments belonging to the same image are not linked. We consider also their timestamps, thus segments not belonging to two images with successive timestamp are not linked. Therefore only segments belonging to two successive timestamps are linked forming an acyclic directed graph. The set of edges, $E_{o^{*}}$, is defined as follows:

$$
E_{o^{*}}=\left\{\left(o^{i}, o^{j}\right) \mid o^{i} \in O_{t} \cap V_{o^{*}}, o^{j} \in O_{t+1} \cap V_{o^{*}}, \operatorname{Pix}\left(o^{i}\right) \cap \operatorname{Pix}\left(o^{j}\right) \neq \emptyset\right\}
$$

Where:

- $O_{t}$ and $O_{t+1}$ are the sets of segments corresponding respectively to the images $I_{t}$ and $I_{t+1}$

- $o^{i}$ and $o^{j}$ are segments of the images $I_{t}$ and $I_{t+1}$ respectively

- $\operatorname{Pix}\left(o^{i}\right)$ is the set of pixels covered by the segment $o^{i}$

- $\operatorname{Pix}\left(o^{j}\right)$ is the set of pixels covered by the reference object $o^{j}$ 


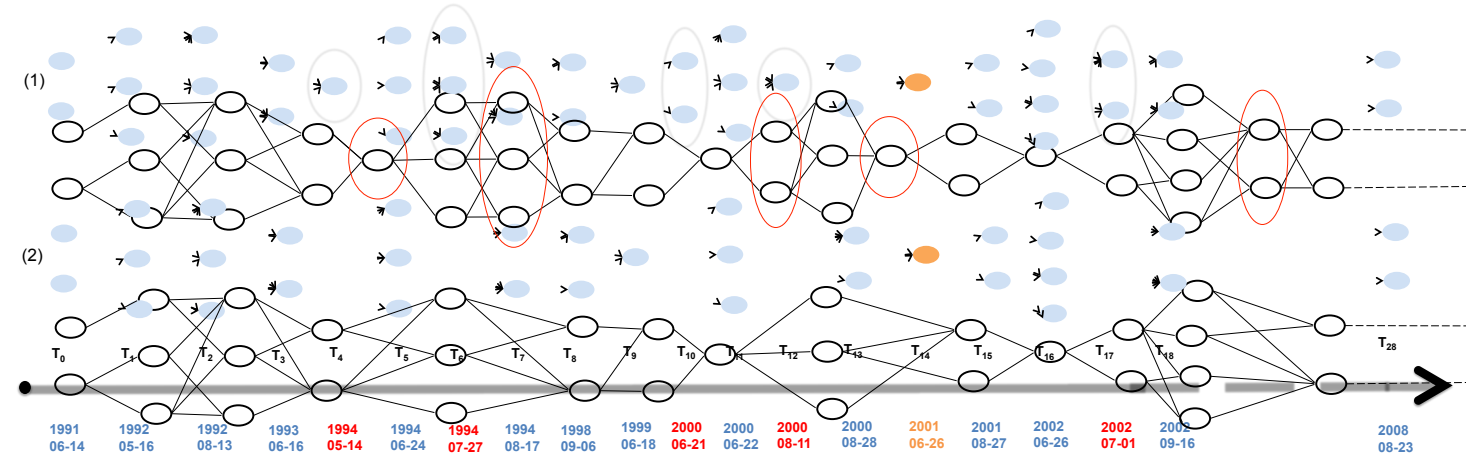

Figure 9: Evolution graph example constructed in the Lower Aude Valley using a reference object selected in the satellite image acquired on 26-06-2011.

Figure 9 shows an example of two evolution graphs $(1,2)$ corresponding to the same reference object. The graph (1) is constructed using all the satellite images of the time series while graph (2) is built considering only the images satisfying the time gap constraint of two months between their timestamps.

We can observe that a graph can be organized by timestamps from left to right. The time line contains: the orange timestamp which is that of reference object, the blue timestamps who are those of the considered images in the evolution graph construction step and the red ones standing for the timestamps of the discarded images. The graph (1) points out the discarded timestamps which do not appear on the final evolution graph (2).

Each timestamp in the time line corresponds to a set of segments in the evolution graph. These timestamps reference to the image in which the segments were identified. As shown in Figure 9 , only the segments corresponding to two successive timestamps are linked. Note that, in the evolution graph, segments correspond to nodes and links correspond to edges.

\subsection{Graph Clustering}

The graph clustering involves the transformation of the evolution graphs into synopsis. The synopsis summarize their information and allows us to compute the distance between them. The distance between two evolution graphs is the distance between their respective synopsis. Considering the distance between each pair of synopsis, a distance matrix is generated. Using the distance matrix, we apply a clustering algorithm that identifies the different groups among the evolution graphs with the aim to distinguish different evolution behaviours.

Figure 10 summarizes the synopsis generation. A synopsis is defined as a sequence of generated nodes, $\left(\widetilde{O_{1}}, \widetilde{O_{2}}, \ldots, \widetilde{O_{t}}, \widetilde{O_{t+1}}, \ldots, \widetilde{O_{T}}\right)$, it contains as many nodes as images used to built the corresponding evolution graph. Each generated node $\widetilde{O_{t}}$ is obtained by a weighted aggregation of the radiometric values of the nodes with the timestamps $t$. This radiometric values are computed as follow:

$$
\operatorname{Info}\left(\widetilde{O_{t}}\right)=\frac{\sum_{o_{t} \in V_{o^{*}}}\left|\operatorname{Pix}\left(o_{t}\right)\right| \operatorname{Info}\left(o_{t}\right)}{\sum_{o_{t} \in V_{o^{*}}}\left|\operatorname{Pix}\left(o_{t}\right)\right|}
$$

The node $o_{t}^{i}$ in the evolution graph (Figure 10 represents a segment, where $t$ is the image timestamp and $i$ is the segment identifier. The evolution graph, in Figure 10 is composed of four groups of segments $\left(\left\{\left\{o_{1}^{1}, o_{1}^{2}\right\},\left\{o_{2}^{1}, o_{2}^{2}\right\},\left\{o_{3}^{1}\right\},\left\{o_{4}^{1}, o_{4}^{2}\right\}\right\}\right)$. A synopsis is defined as a sequence of averaged segments, one segments per timestamps (image) i.e., each group of segments in the evolution graph is transformed into one averaged $\operatorname{object}\left(\left(\widetilde{O_{1}}, \widetilde{O_{2}}, \widetilde{O_{3}}, \widetilde{O_{4}}\right)\right)$.

More in detail, the segments of each evolution graph are aggregated per image. They are first weighted by their size to give more importance to bigger segments, then averaged to create only one segment. The segments in the evolution graph are considered as a vector of features (spectral bands and radiometric 


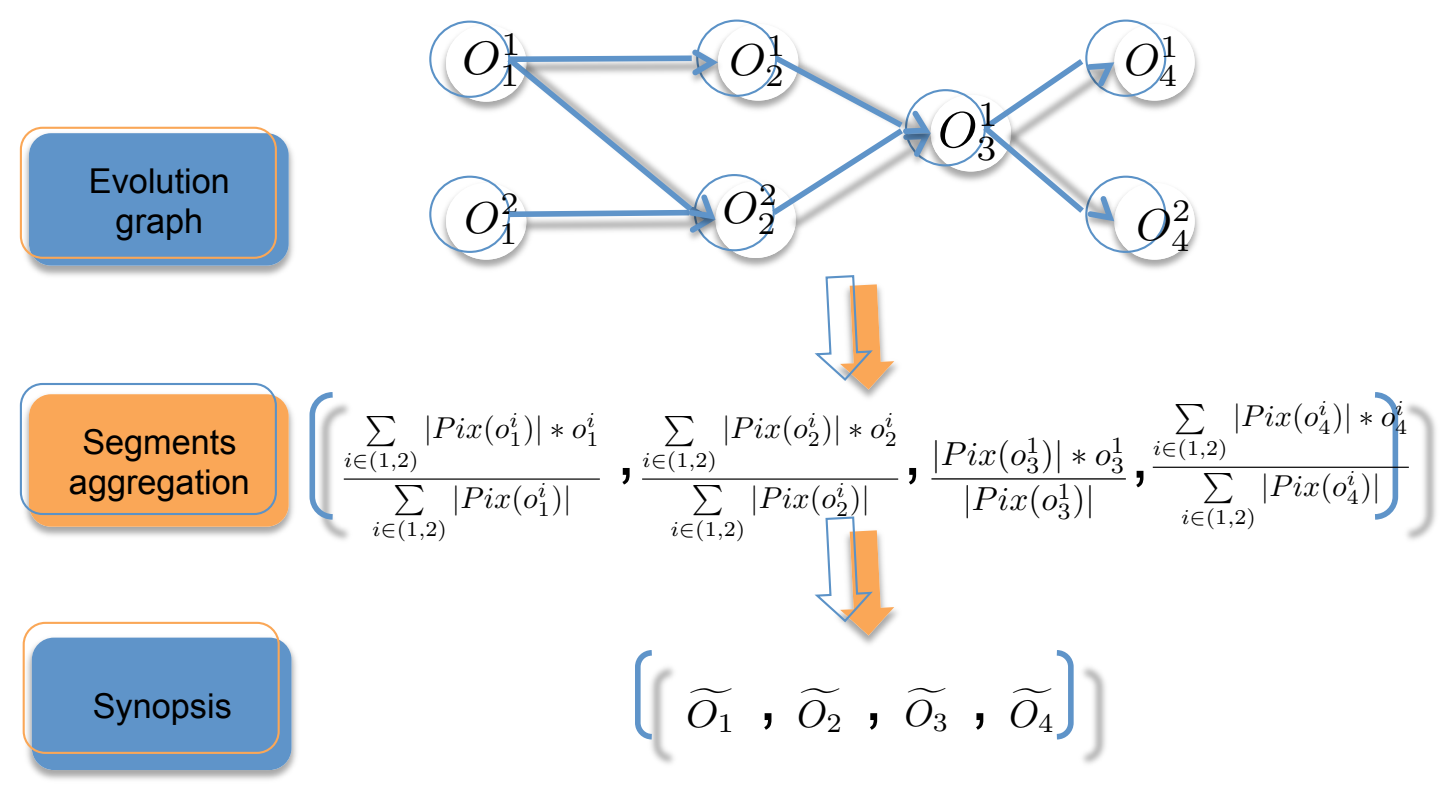

Figure 10: The procedure to build a synopsis of an evolution graph that aggregates together the set of segments of each satellite image.

indices: green, red, near infared, NDVI, NDWI, BI, CI and SAVI ). Each vector/segment is first weighted by its number of pixels. Then, it is averaged to create new vector/segment.

Once the evolution graphs are transformed into a synopsis. The next step is the distance computation. The distance is computed for each pair of synopsis. Given two evolution graphs $G_{1}$ and $G_{2}$, we define their corresponding synopsis $s y n_{1}$ and $s y n_{2}$, then we compute the distance between them as following:

$$
\operatorname{dist}\left(\operatorname{syn}_{1}, \operatorname{syn}_{2}\right)=\operatorname{DTW}\left(\operatorname{syn}_{1}, \operatorname{syn}_{2}\right)
$$

To estimate the distance between the synopsis, we use the Dynamic Time Warping (DTW) distance measure [30]. This distance measure allows to estimate the distance between two time series of different sizes and find their optimal alignment. It is a time-designed distance measure able to gather time-distorted sequences with temporal shifts 31 . Considering these proprieties, DTW is adapted to our study case. In fact, the number of satellite images considered for each site is different due to noisy and missing information (i.e. cloudy images). Therefore, the resulting synopsis can have different lengths. Note also that, for the same study site, the timestamps of evolution graph can differ from each other due to the time gap constraint.

Give two time series $C=\left(c_{1}, c_{2}, \ldots, c_{n}\right)$ and $Q=\left(q_{1}, q_{2}, \ldots, q_{m}\right)$, of length $n$ and $m$ respectively $(n \neq m)$. To compute the distance between this two time series using the dynamic time warping (DTW) measure, a matrix $D$ of size $n * m$ is constructed as follows:

$$
D\left(c_{l}, q_{r}\right)=\theta\left(c_{l}, q_{r}\right)+\min \left\{\begin{array}{l}
D\left(c_{l-1}, q_{r-1}\right) \\
D\left(c_{l}, q_{r-1}\right) \\
D\left(c_{l-1}, q_{r}\right)
\end{array}\right.
$$

Where:

- $\theta$ is a distance measure (Euclidian distance is the most one used)

The matrix is initialized computing the first element $\left(D\left(c_{1}, q_{1}\right)\right)$. The other elements are then computed using the minimum between the left, upper or diagonal one. The distance between the two series is given by the last element: 
the synopsis into homogeneous clusters. Each cluster groups similar synopsis. The clustering process consider the evolution graphs of the different sites and allows discovering similarities among them. The clustering is performed using the hierarchical agglomerative algorithm [32]. This clustering algorithm creates a dendrogram which is a tree-level structure, where each level contains a partition set.

The hierarchical agglomerative algorithm begins with each point in its own cluster and progressively joins the closest clusters until only one cluster remains. Thus, we result in different clustering solutions with different cluster number which allows the expert to choose a clustering solution considering different granularity.

We remind that also in 33 the authors perform evolution graphs clustering but, conversely to them, in our scenario: i) we consider multi-annual time series, ii) we jointly analyse several sites at the same time and iii) we deal with time series with variable length.

\section{6. $\sigma_{1}$ and $\sigma_{2}$ setting}

The described approach use three parameters:

- $\alpha$ allows to select relevant reference objects.

- $\sigma_{1}$ and $\sigma_{2}$ avoid to associated noisy segments to an evolution graph.

The threshold $\alpha$ allows to select a subset of reference objects while minimizing the overlapping among them. The idea is to select segments that cover as much as possible the study area while minimizing the overlapping. While $\sigma_{1}$ and $\sigma_{2}$ avoid to select irrelevant segments in the evolution graph construction i.e., select only the segments that cover the reference object spatial extent on the time series.

To select the values of the three parameters we consider the coverage and overlapping of the evolution graphs. The evolution graph coverage defines the area covered by all the segments of all the constructed evolution graphs while, the evolution graphs overlapping defines the area covered by at least two evolution graphs.

To fix the appropriate parameters values, we varied them in range $[0,1]$ generating different combinations. Then, we use the different combinations to construct the evolution graphs and report their coverage and overlapping. Considering the obtained coverage, we fix a threshold, named $\tau$, for the minimum acceptable coverage. Among the combinations that satisfy the threshold, we select the one with the minimum overlapping rate.

\subsection{Validation of the results}

Once the reference objects are selected, their evolution are analysed and grouped in different clusters using the Hierarchical Agglomerative Clustering (HAC) algorithm. The clusters involve the graphs evolving similarly. Each cluster highlights a different behaviour pattern.

To evaluate our framework, the evolution graphs are classified by the field expert in nine land cover classes as described in section 2.4. Then, the identified classes are compared to the clustering results using two evaluation measures: the Normalized Mutual Information (NMI) and the Adjusted Rand Index (ARI) 34 .

To define the NMI and ARI, Lets consider a clustering result $\mathbf{C}=\left\{C_{1} \ldots C_{J}\right\}$ with $J$ clusters and an expert classification $\mathbf{P}=\left\{P_{1} \ldots P_{I}\right\}$ with $I$ classes. We note $n$ the numbers of entities to analyse.

The NMI is a clustering validity index that measures the information shared between the generated clusters and the identified classes. This metric is independent from the number of clusters and ranges between 0 and 1 , the higher the value the more the clustering grouping and the expert classification partition agree. It is computed considering each pair of clusters and classes as follows:

$$
\mathbf{N M I}(\mathbf{C}, \mathbf{P})=\frac{\sum_{i=1}^{I} \sum_{j=1}^{J} x_{i j} \log \frac{n x_{i j}}{x_{i} x_{j}}}{\sqrt{\sum_{i=1}^{I} x_{i} \log \frac{x_{i}}{n} \sum_{j=1}^{J} x_{j} \log \frac{x_{j}}{n}}}
$$


Where:

- $x_{i j}$ is the cardinality of the set of entities that belong to cluster $C_{j}$ and class $P_{i}$

- $x_{j}$ is the number of entities in the cluster $C_{j}$

- $x_{i}$ is the number of objects in the class $P_{i}$

The ARI measures the agreement between two partitions by considering all pairs of entities and counting pairs that are assigned in the same or different cluster in two partitioning. We note $a$ the number of entity pairs belonging to the same cluster in $C$ and the same class in $P$, the ARI is computed using the following equation:

$$
\mathbf{A R I}(\mathbf{C}, \mathbf{P})=\frac{a-E[a]}{\max (a)-E[a]}
$$

Where:

- $E[a]$ represents the expectation value of $a$

- $\max (a)$ represents the maximum value of a

$E[a]$ and $\max (a)$ are computed using the following equations:

$$
\begin{gathered}
E[a]=\frac{\pi(C) \cdot \pi(P)}{n(n-1) / 2} \\
\max (a)=\frac{1}{2}(\pi(C)+\pi(P))
\end{gathered}
$$

Where:

- $\pi(C)$ denote the number of object pairs that belong to the same cluster in $\mathbf{C}$

- $\pi(P)$ denote the number of object pairs that belong to the same class in $\mathbf{P}$

The rand index lies between 0 and 1. When the two partitions agree perfectly, the ARI is equal to 1 . The obtained results are reported and discussed in the next section.

\section{Experimental Results and Discussion}

In this section, we present and discuss the results of our analysis. First, we extract the evolution graphs from the study areas adopting the proposed approach. Then, we perform the clustering analysis on the evolution graphs obtained from the three study areas. Finally, we present qualitative results on the obtained clusters.

\subsection{Parameters Setting}

Our framework needs as input three parameters $\left(\alpha, \sigma_{1}\right.$ and $\left.\sigma_{2}\right)$ that allow us to select informative and non-redundant sets of reference objects from the SITS data. With the aim to choose the parameter combination that maximize the coverage, we vary their range in the interval $[0,1]$ with a step of 0.1 . For each combination, the set of evolution graphs is constructed. Associated to each set of evolution graphs, we can compute the coverage and the overlapping ratio. Figure 11 reports the coverage for the three study sites. We discretize the coverage ratio in bins (the histogram bins range between $0 \%$ and $100 \%$ with a step of $5 \%$ ). Each bin represents how many times a parameter combination produces a set of evolution graphs with that particular coverage. 


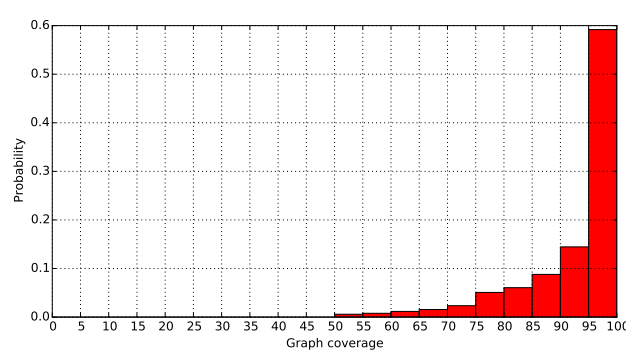

(a)

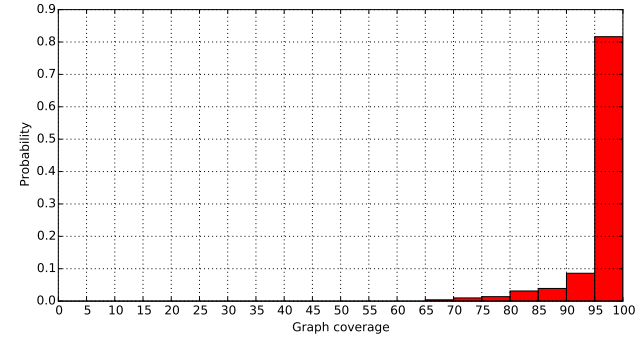

(b)

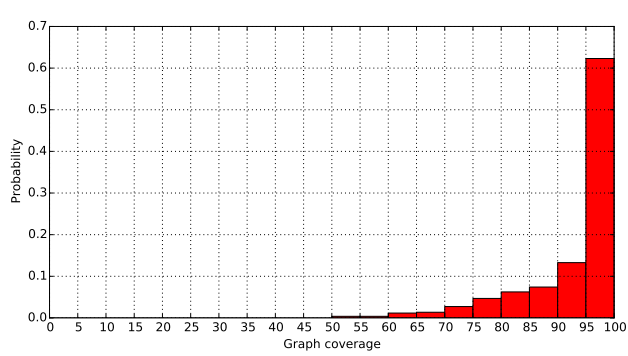

(c)

Figure 11: The probability of the evolution graphs coverage rate of the three sites: (a) Lower Aude Valley, (b)MMCA and (c) Pic Saint Loup.

Figure 11 reports the evolution graphs coverage for the Lower Aude Valley (Figure 11(a), the MMCA (Figure 11(b) and the Pic Saint Loup (Figure 11(c))

Once the results are analysed, we select $85 \%, 90 \%, 90 \%$ as coverage threshold for the Lower Aude Valley, the MMCA and the Pic Saint Loup respectively. As observed in Figure11, more than $50 \%$ of the combinations cover more than $95 \%$ of the study area, despite that the fixed thresholds are inferior to $95 \%$. This is due to the high overlapping rate of the generated graphs by these combinations. The retained combination generates a set of evolution graphs covering as much as possible the study areas while minimizing the overlapping rate.

Using the selected parameters values, we extract 233 reference objects: 67 on the Lower Aude Valley, 87 on the Pic Saint Loup and 79 on the MMCA sites. The selected reference objects result on 233 evolution graphs covering around $89 \%$ of the three study areas. Figure 4 reports the selected parameters values as well as the coverage and overlapping rate of the resulting evolution graphs.

\begin{tabular}{|l|l|l|l|l|l|l|}
\hline & $\alpha$ & $\sigma 1$ & $\sigma_{2}$ & $\begin{array}{l}\text { Evolution } \\
\text { graph }\end{array}$ & $\begin{array}{l}\text { Evolution } \\
\text { graphs } \\
\text { coverage }\end{array}$ & $\begin{array}{l}\text { Evolution } \\
\text { graphs } \\
\text { overlapping }\end{array}$ \\
\hline Lower Aude Valley & 0.5 & 0.9 & 0.7 & 67 & 88.27 & 26.02 \\
\hline MMCA & 0.4 & 0.9 & 0.8 & 79 & 90.29 & 22.71 \\
\hline Pic Saint Loup & 0.3 & 0.9 & 0.8 & 87 & 90.80 & 30.3 \\
\hline
\end{tabular}

Table 4: The selected parameters values and the numbre of resulting evolution graphs with their coverage and overlapping rate on each study area.

\subsection{Clustering Results}

The generated evolution graphs are grouped with the aim to characterize common behaviour among the different spatio-temporal phenomena. To this end, we use the hierarchical agglomerative clustering with 
Author-produced version of the article published in

International Journal of Applied Earth Observation and Geoinformation, 2019, N 74, p. 103-119

The original publication is available at

http://www.sciencedirect.com/science/article/pii/S0303243418304781

Doi: 10.1016/j.jag.2018.07.014

average linkage. This algorithm creates a hierarchy of partitions with different number of clusters. Among the different level of the hierarchy, we choose the solution that involves 20 clusters.

The clustering algorithm works on the evolution graphs features (the spectral bands and the spectral indices). With the aim to understand the influences of the different feature groups, we cluster the evolution graphs considering two different scenario: (i) a clustering performed considering only the spectral bands and (ii) a clustering performed considering both spectral bands and spectral indices.

Figure 12 illustrates the obtained results from the analysis of the evolution graphs using the three spectral bands: Green, Red and Near Infrared. Whereas, figure 13 illustrates the results obtained combining the spectral bands and the spectral indices: NDVI, NDWI, BI, CI and SAVI.
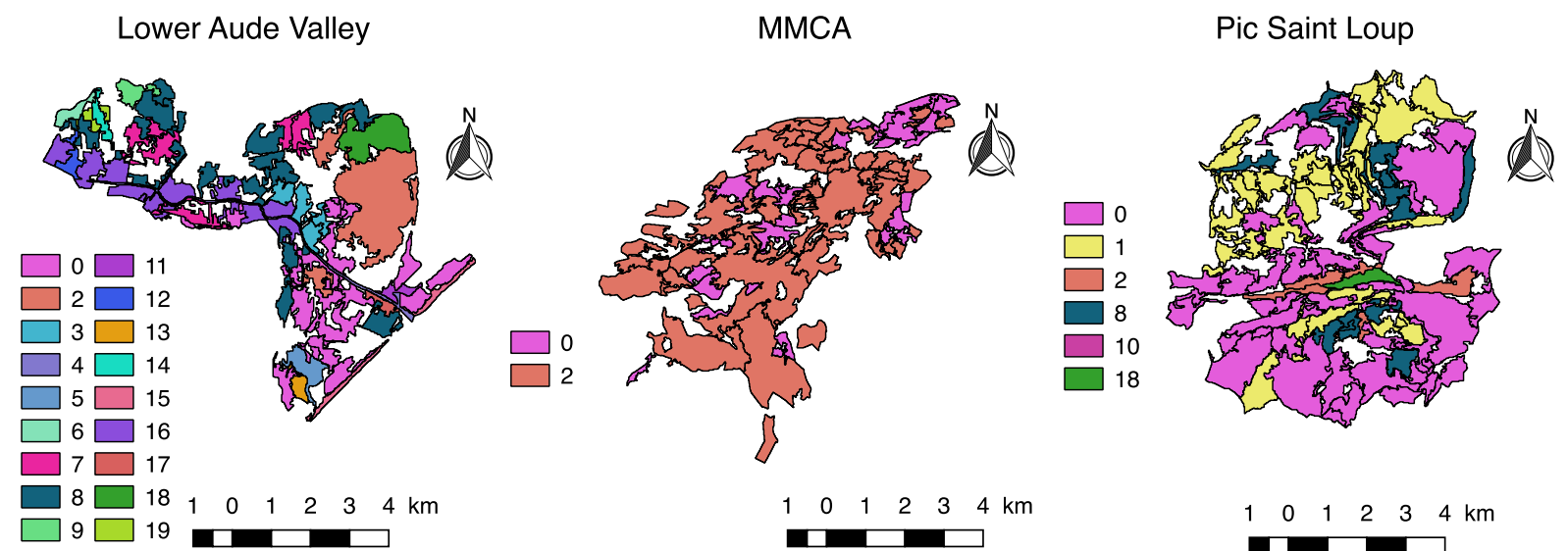

Figure 12: The distribution of the reference objects in the 20 clusters on the three sites using the spectral bands: Green, Red and Near Infrared.

Lower Aude Valley

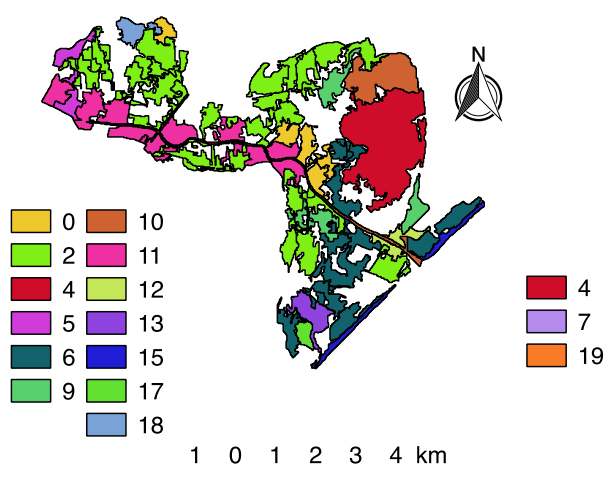

0
MMCA

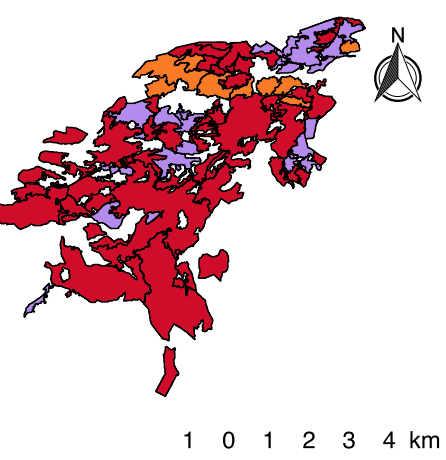

$\begin{array}{llllll}0 & 1 & 2 & 3 & 4 \\ k n & 0 & 0 & 0 & 0\end{array}$
Pic Saint Loup
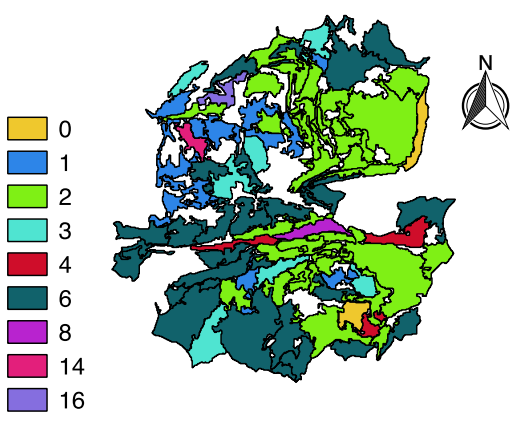

$\begin{array}{lllllll}1 & 0 & 1 & 2 & 3 & 4 & \mathrm{~km}\end{array}$

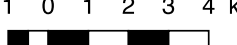

Figure 13: The distribution of the reference objects in the 20 clusters on the three sites using both the spectral bands and the spectral indices: Green, Red, Near Infrared, NDVI, NDWI, BI, CI and SAVI .

A. Lower Aude Valley 
For the Lower Aude Valley (Figure 12), the evolutions of the reference objects are grouped in 18 clusters. The Water area class corresponds to the clusters 4,5 and 18. The class Wet area is represented by the clusters 11 and 13 while the class Beach grouped in the cluster 15 . The class Vineyard is also grouped in the clusters 6, 12 and 16, this partitioning in different clusters is explained by the phenological stage of the observed vineyard. More in detail, depending on the stage of their development, the radiometric values of the vineyards differ, which makes their discrimination possible. However, the clusters 0, 7 and 8 group the different vegetation canopy classes (Vineyard, Shrubby vegetation and Crop) and the wetland (Water area and Wet area), The cluster 2 groups the aquatic vegetation among the two classes Wet area and Water area. The class Crop is identified on the clusters 3,14 and 17 .

In the second case (Figure 13), the radiometric information allows to distinguish the different classes among the Lower Aude Valley, The class Water area is grouped in the clusters 10, 13 and 4 which stands for the aquatic vegetation. The class Beach is represented by the cluster 15. Also the class Wet area is grouped in the clusters 9 and 17. The vegetation is grouped in the cluster 0 (Crop and Vineyard). The clusters 2, 6 and 12 group the classes Crop, Vineyard, Wet area and Shrubby vegetation that show similarities in their radiometric reflectance values on the considered period.

B. The Mountain of the Moure and Causses of Aumelas

Despite the number of clusters (20), we identified 2 clusters on the MMCA site (Figure 12). The cluster 0 groups the vegetation canopy (Crop, Shrubby vegetation, Vineyard and open space with no or few vegetation). These reference objects evolutions are characterized by high reflectance values in all bands. When the cluster 2 groups the Forest and the Shrubby vegetation classes which are characterised by low reflectance values in the visible bands (Green and Red) and hight values in the Near Infrared.

Considering both spectral bands and the spectral indices, we identify 3 clusters in the MMCA (Figure 13p. The cluster 19 is specific to the class Forest, the cluster 4 groups the classes Forest and Shrubby vegetation. The cluster 7 groups the Vineyard and Shrubby vegetation classes. The NDVI and SAVI indices allow to identify the density variation of the vegetation canopy.

\section{Pic Saint Loup}

In The Pic Saint Loup, we detect 6 clusters (Figure 12). The class Forest is well discriminate on the clusters 2 and 8 . The cluster 18 corresponds also to Forest, however the shaded side of the Pic Saint Loup mountain affect its radiometric properties. The cluster 1 which groups the vegetation canopy (Crop, Shrubby vegetation and Vineyard). Cluster 0 groups the class Forest and Shrubby vegetation which have high reflectance in the NIR band, this fact explains that these two classes are grouped together.

With the use of the spectral indices, we identified 9 clusters in Pic Saint Loup site (Figure 13). Cluster 2, 3 and 6 group the classes Shubby vegetation and Forest that evolve similarly. The clusters 0,4 and 8 group the Forest class. Regarding classes Crop and Vineyard, they are associated to cluster 1 and 14.

The main objective of this work is to perform an object-oriented analysis on multi-annual SITS data to underline intra and inter-site similarities among the different study areas. To deal with this goal, we have proposed a method to analyse multi-annual SITS data based on a graph representation. To asses the proposed method, we perform analysis on three study areas. As a result, we identify both: spatio-temporal evolutions specific to an area and and spatio-temporal evolution that are shared among different sites.

Considering the clustering performed only on spectral bands (Figure 12), the clusters 0 and 2 group spatio-temporal entities of the three study areas. Those phenomenon show similar temporal behaviours. 
the Pic Saint Loup but, it corresponds to Wet area and vegetation classes on the Lower Aude Valley. The Wet area class is grouped with the vegetation classes because they share a close radiometric response.

Clusters 8 and 18 group together entities of the Pic Saint Loup and the Lower Aude Valley. Cluster 18 corresponds to water on the Lower Aude Valley and shadow areas on the Pic Saint Loup. The grouped phenomenon in cluster 18 have a different radiometric response in the Near Infrared band, but they show similarities in the Red and Green bands. On the other hand, cluster 8 corresponds to vegetation on the two sites, it groups Vineyard, Crop and Wet land objects on the Lower Aude Valley and Forest, Shrubby vegetation and Crop classes on the Pic Saint Loup.

When combining the spectral bands with the spectral indices (Figure 13), similar phenomenon among the three study areas are grouped together in cluster 4 . Cluster 4 corresponds to Forest in the Pic Saint Loup, it involves Forest and Shrubby vegetation coming from the MCCA site while, for the Lower Aude Valley, it corresponds to aquatic vegetation (Water area). The fact that these classes are gathered in the same group is explained by their similarity in the NDVI response.

Regarding the Lower Aude Valley and the Pic Saint Loup, the clusters 2 and 6 group similar spatiotemporal entities together. Cluster 2 corresponds to Forest in the former site and groups the classes: Crop, Vineyard and Wet area in the latter site. The spatio-temporal entities in the cluster 2 have similar NDVI and SAVI evolution. Concerning cluster 6, it groups spatio-temporal entities of theShrubby vegetation class on the Pic Saint Loup and Wet area class on the Lower Aude Valley. These two classes are grouped together as they have a similar radiometric evolution considering all the set of bands and indices.

To assess the performance of our clustering approach, we compared the clustering result to the classification supplied by the field expert. We compute the NMI and the ARI between these two partitions. More in detail, we repeat this comparison four times, one for each of the site independently (in the context of intra-site analysis) and once considering the simultaneous clustering of the full set of study areas (in the context of the inter-site analysis). The analysis are performed considering two different configurations: only the raw bands (Figure 12) and the combination of raw bands and radiometric indices (Figure 13). The results are reported in Table 5

Considering the NMI results, despite some confusion in the Pic Saint Loup, we note that the method performs generally better using the spectral bands combined with the indices than using only the spectral bands. Considering the ARI results, they show that including the spectral indices in the analysis enhances the clustering process excepting for the Lower Aude Valley in which better results are achieved using only the spectral bands.

\begin{tabular}{|l|l|l||l|l|}
\hline \multirow{2}{*}{} & \multicolumn{2}{|l||}{ Spectral bands } & \multicolumn{2}{l|}{$\begin{array}{l}\text { Spectral bands } \\
\text { and indices }\end{array}$} \\
\cline { 2 - 5 } & ARI & NMI & ARI & NMI \\
\hline \hline Lower Aude Valley & $\mathbf{0 . 2 5}$ & 0.258 & 0.193 & $\mathbf{0 . 2 9 2}$ \\
\hline MMCA & 0.104 & 0.151 & $\mathbf{0 . 2 1 8}$ & $\mathbf{0 . 2 3 8}$ \\
\hline Pic Saint Loup & 0.164 & $\mathbf{0 . 2 6}$ & $\mathbf{0 . 2 2 3}$ & 0.211 \\
\hline Intra-sites & 0.152 & 0.236 & $\mathbf{0 . 1 5 9}$ & $\mathbf{0 . 2 4 6}$ \\
\hline
\end{tabular}

Table 5: Normalized Mutual Information and Adjusted Rand Index results obtained using: i) the spectral bands ii) the spectral bands plus the radiometric indices on the study areas.

\subsection{Evolution Graphs Analysis Results}

With the aim to inspect the spatio-temporal evolution extracted by our approach, we report in Figures 14 . 18 some examples of evolution graphs. Such examples depict representative evolution graphs belonging to cluster 4 and 6 .

We avoid to draw the whole evolution graphs since they are composed by more than twenty timestamps. To improve the readability we select only some timestamps (five) for each evolution graph. The colour of the segments represents their radiometric values. Thus, the change of the colours indicates the evolution of 
Author-produced version of the article published in

International Journal of Applied Earth Observation and Geoinformation, 2019, N 74, p. 103-119

The original publication is available at

http://www.sciencedirect.com/science/article/pii/S0303243418304781

Doi: 10.1016/j.jag.2018.07.014

the considered segments. We visualize the satellite images segments in false-colour. Adopting this choice, the vegetation canopy will appear in red.

Figure 17 and Figure 18 show two evolution graphs belonging to the Lower Aude Valley and the Pic Saint Loup respectively. Both evolution graphs are grouped in cluster 6 and define a reference object covered by shrubby vegetation. As we know the evolution of shrubby vegetation is progressive and it remains constant most of the time. This is why the colour of the segments remains mostly unchanged in all the timestamps.

Figures 14, 15 and 16. show three evolution graphs corresponding to the Lower Aude Valley, the MMCA and the Pic Saint Loup respectively. They are grouped together in cluster 4 . The two last evolution graphs (Figure 15 and Figure 16) represent forest areas which are characterized by a dense vegetation canopy, thus the satellite images are characterized by a darkened red colour. In addition, the forest cover is not influenced by seasonal change, thus the radiometric values of those areas remain unchanged. Figure 14 illustrates an evolution graph of a water area covered by aquatic vegetation, the water area appears in darkened blue while the vegetation appear in red. This combination of radiometric values is quiet similar to the forest cover, thus these three evolution graphs are grouped together.

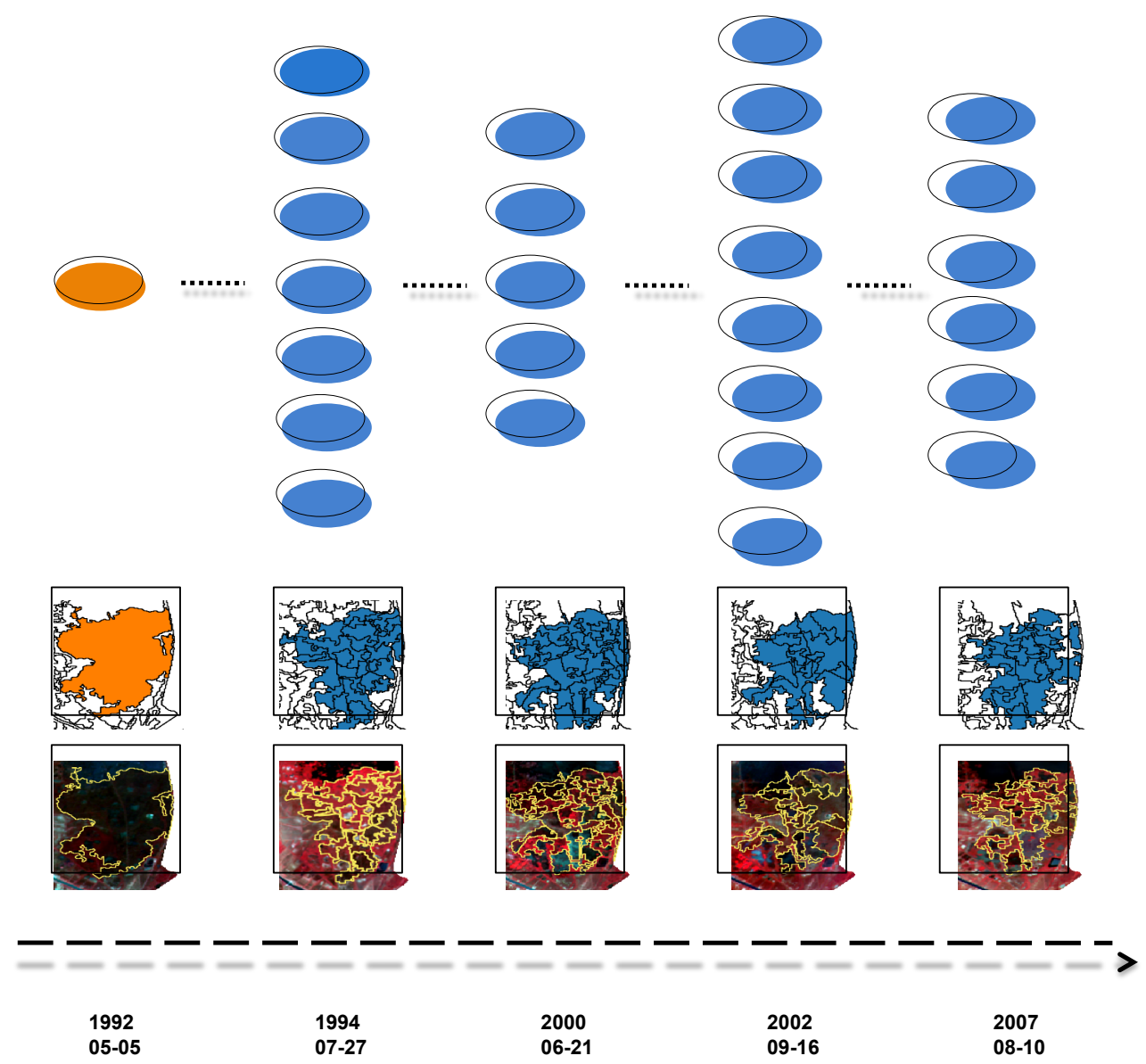

Figure 14: Evolution graph example of the Lower Aude Valley describing the dynamic of a water area covered by aquatic vegetation grouped in the cluster 4 .

To sum up, we analysed the clustering results for the three study areas considering only raw images (spectral bands) and spectral bands plus derived spectral indices. When only raw bands are employed, we note that this information is not enough to distinguish among the different land cover classes. Otherwise, 
Author-produced version of the article published in

International Journal of Applied Earth Observation and Geoinformation, 2019, N 74, p. 103-119

The original publication is available at

http://www.sciencedirect.com/science/article/pii/S0303243418304781

Doi: 10.1016/j.jag.2018.07.014

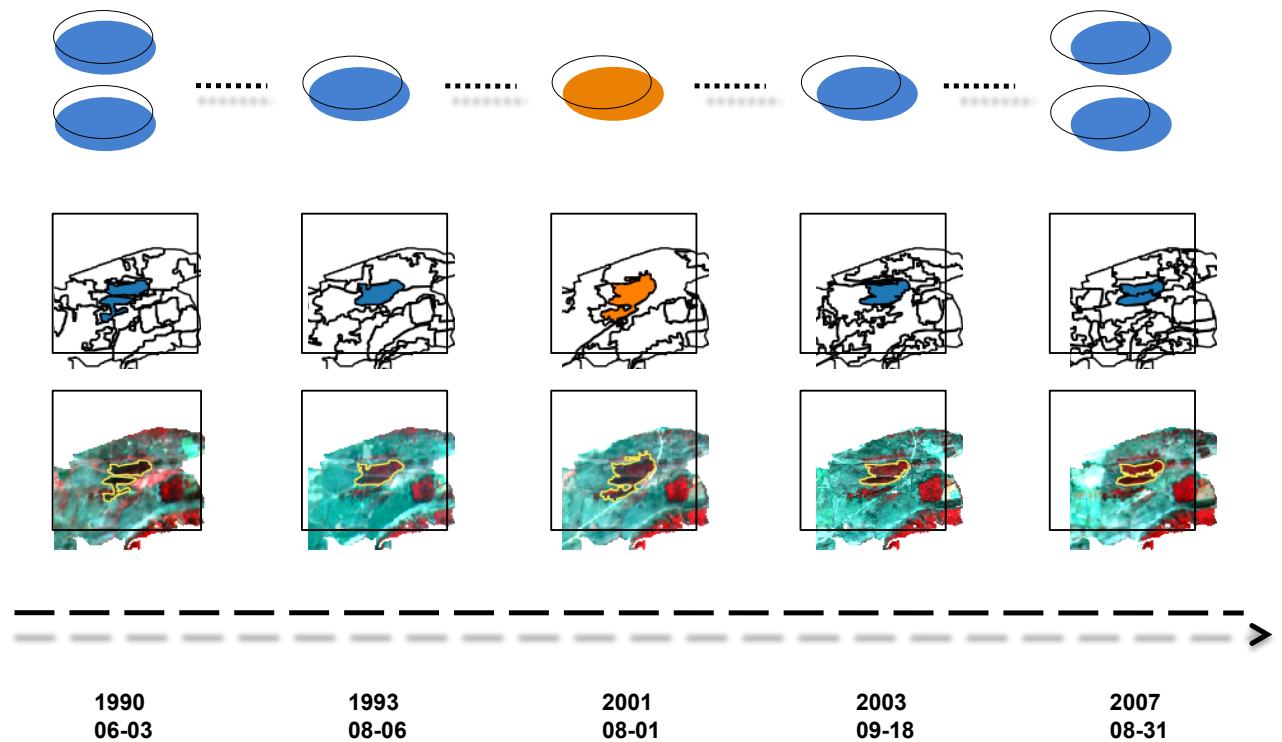

Figure 15: Evolution graph example of the $M M C A$ describing the dynamic of a forest area grouped in the cluster 4.
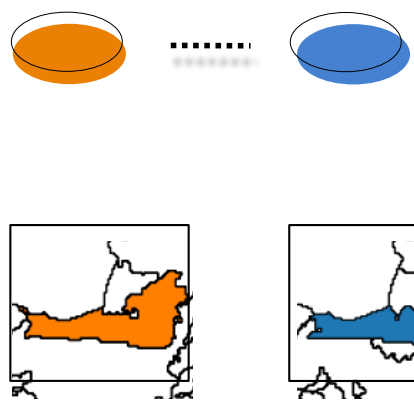

$\cdots+\cdots$

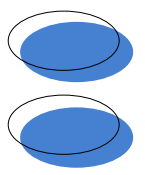

........

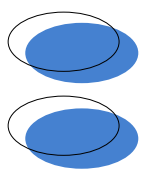

........
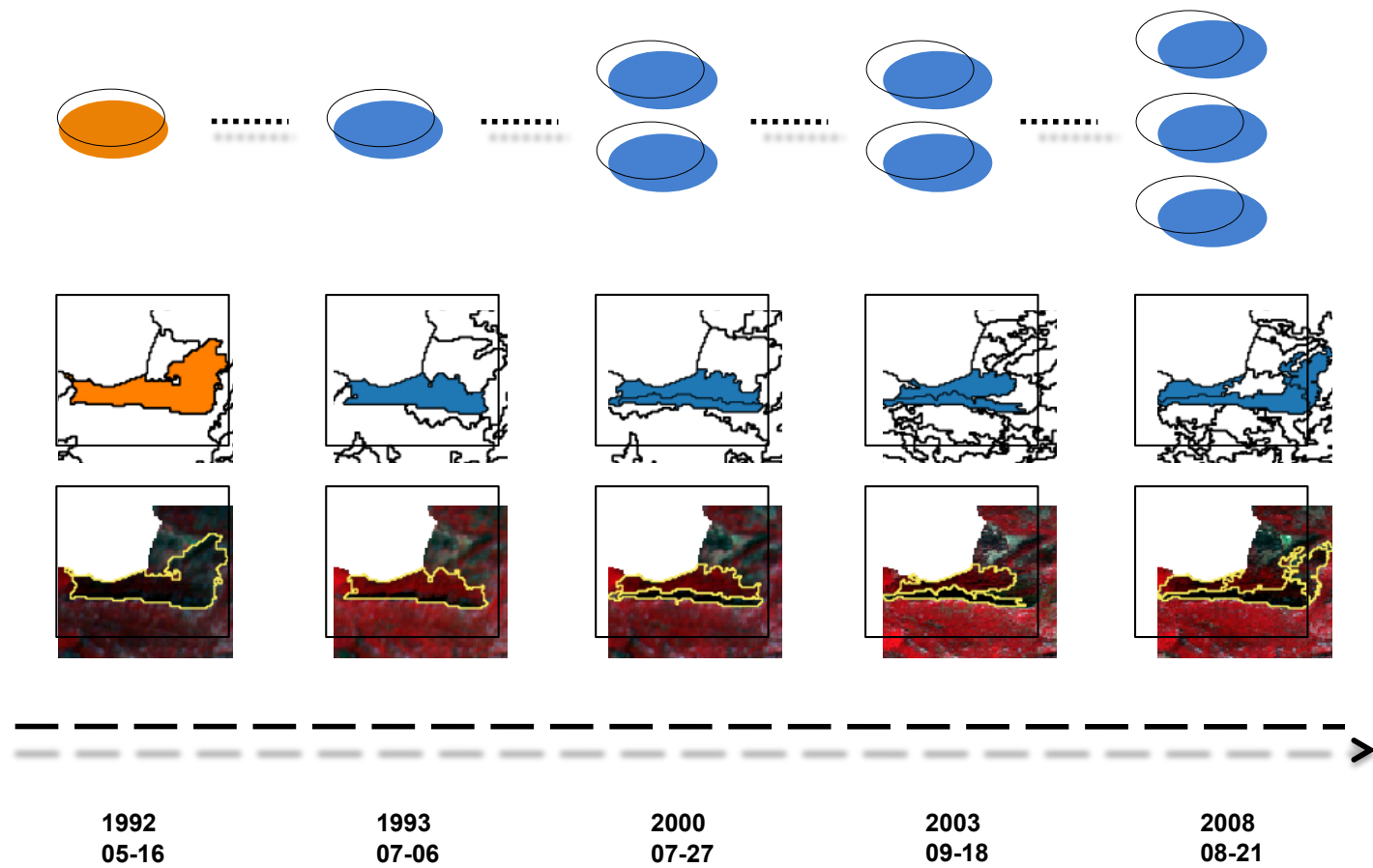

Figure 16: Evolution graph example of the Pic Saint Loup describing the dynamic of a forest area grouped in the cluster 4.

the use of spectral indices induces an improvement of the clustering results.

The introduced method allowed us to perform intra-site and inter-site analysis to explore simultaneously 
Author-produced version of the article published in

International Journal of Applied Earth Observation and Geoinformation, 2019, N 74, p. 103-119

The original publication is available at

http://www.sciencedirect.com/science/article/pii/S0303243418304781

Doi: 10.1016/j.jag.2018.07.014

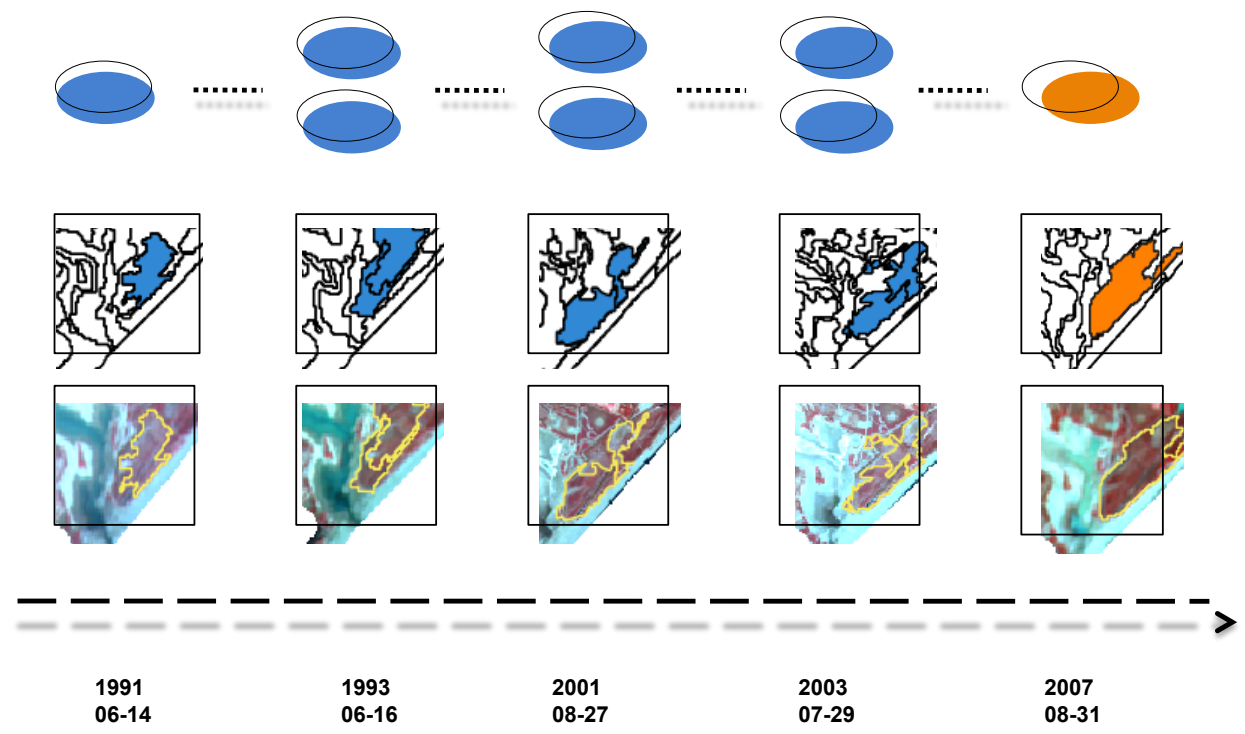

Figure 17: Evolution graph example of the Lower Aude Valley describing the dynamic of an area covered by shrubby vegetation grouped in the cluster 6.

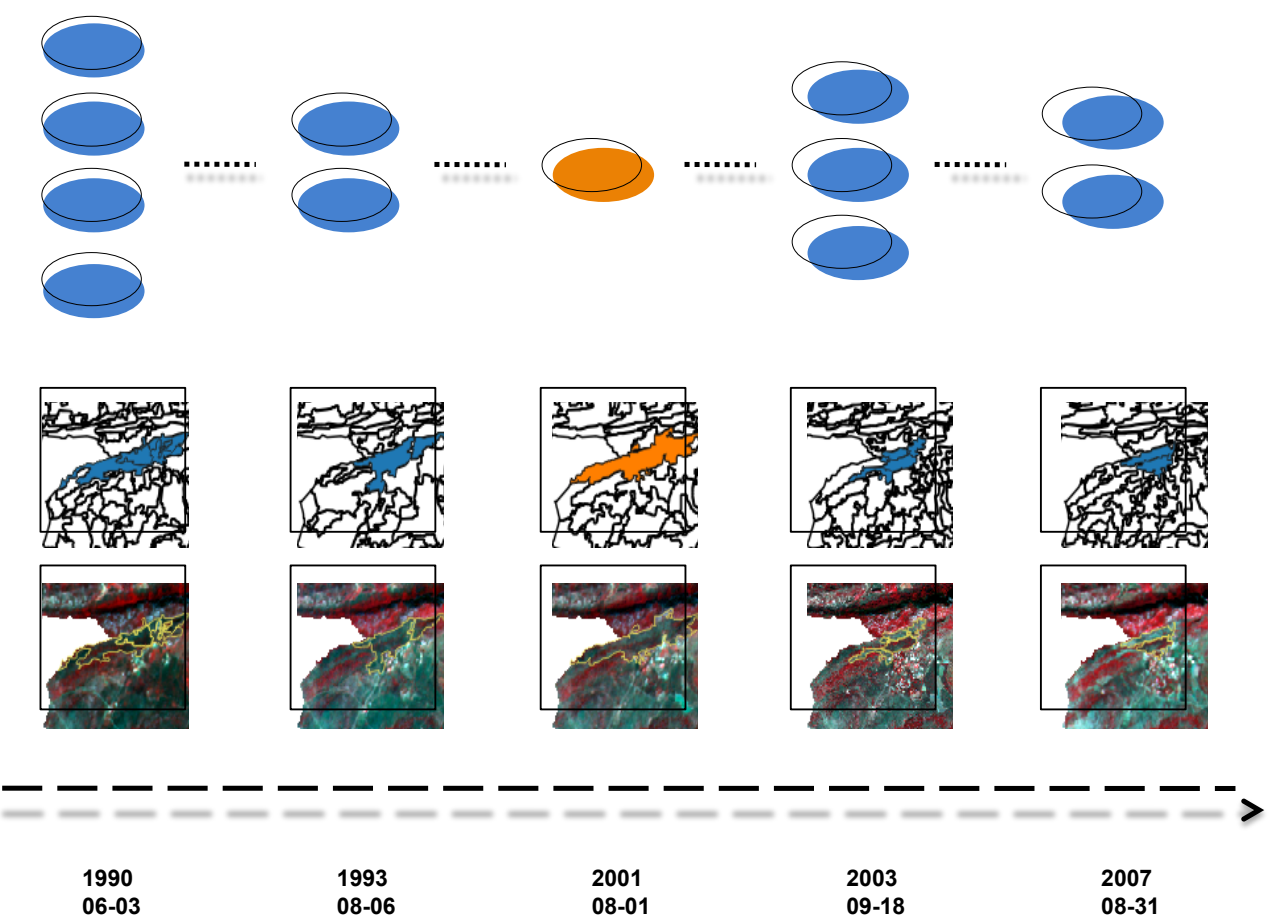

Figure 18: Evolution graph example of the Pic Saint Loup describing the dynamic of an area covered by shrubby vegetation grouped in the cluster 6 .

the temporal evolution of several study areas. Operationally, this method can be exploited by geographers to 
explore new areas making the connection with spatially closed zone on which previous knowledge is already available.

They can derive the characteristics of an unexplored area from the explored one by applying our framework on them. It offers a tool to inspect remotely study areas, thus saving time and human effort. It also allows the experts to understand phenomena evolution by comparing them to those already depicted. Note also that our approach first processes each study area independently and then combine them. Thus, it is adapted to parallel processing and can handle large zones involving several areas by decomposing the analysis in a divide-et-impera strategy.

\section{Conclusion}

This work has introduced an object-oriented approach to analyse multi-annual time series spanning over multiple study areas. The proposed method has demonstrated its effectiveness on real world data.

The method takes as inputs the satellite images and the corresponding (per image) segmentation. Then, it identifies the reference objects to analyse and illustrate their evolution trough a graph based representation. Finally, the obtained graphs are clustered to identify homogeneous groups that share similar muti-annual dynamic.

We have experimented the proposed method performing both intra and inter-site analysis on three study areas located in the south of France. The involved SITS data describe the study areas over eighteen years from 1990 to 2008. The clustering results have been extensively inspected with the aim to validate the extracted knowledge by means of clustering validity indices as well as in-depth analysis. The experiments have shown that our method extracts representative evolutions describing the study areas. We have also pinpointed its ability to make connections among different study areas. This last point can be particularly helpful if we want to transfer knowledge from an area to another study area. Finally, we have highlighted that using the spectral indices combined with the spectral bands allows to better discriminate spatio-temporal phenomena evolving similarly than using only spectral bands.

As future work, we plan to investigate how to take advantage of the topological and geometric features we can extract from the evolution graphs structure. Furthermore, we also plan to introduce background knowledge in the pipeline to guide the clustering to meet as much as possible the expert expectations. A possible way to do this will be the use of some expert knowledge (i.e. under the form of constraints) to ensure semi-supervision in the current fully unsupervised process.

\section{Acknowledgment}

The authors wish to thank Algerian Ministry of Higher Education and Scientific Research and the French Space Study Center (CNES, Dynamitef 2017 TOSCA) for supporting this research.

\section{References}

[1] J. Knorn, A. Rabe, V. C. Radeloff, T. Kuemmerle, J. Kozak, P. Hostert, Land cover mapping of large areas using chain classification of neighboring landsat satellite images, Remote Sensing of Environment 113 (5) (2009) 957 - 964. doi:https://doi.org/10.1016/j.rse.2009.01.010. URL http://www.sciencedirect.com/science/article/pii/S0034425709000194

[2] A. Mohammed, S. Rusthum, Object-oriented image processing of an high resolution satellite imagery with perspectives for urban growth, planning and development, International Journal of Image Processing, Computer Science Journals 2 (3) (2008) 18-28.

[3] S. Velickov, D. Solomatine, X. Yu, R. Price, Application of data mining techniques for remote sensing image analysis, in: Proc. 4th Int. Conference on Hydroinformatics, USA, 2000.

[4] M. Li, S. Zang, B. Zhang, S. Li, C. Wu, A review of remote sensing image classification techniques: the role of spatiocontextual information, European Journal of Remote Sensing 47 (1) (2014) 389-411. arXiv:https://doi.org/10.5721/ EuJRS20144723, doi:10.5721/EuJRS20144723. URL https://doi.org/10.5721/EuJRS20144723

[5] F. Petitjean, J. Inglada, P. Gançarski, Satellite image time series analysis under time warping, IEEE Trans. Geoscience and Remote Sensing 50 (8) (2012) 3081-3095. doi:10.1109/TGRS.2011.2179050. URL https://doi.org/10.1109/TGRS.2011.2179050 
Author-produced version of the article published in

International Journal of Applied Earth Observation and Geoinformation, 2019, N 74, p. 103-119

The original publication is available at

http://www.sciencedirect.com/science/article/pii/S0303243418304781

Doi: 10.1016/j.jag.2018.07.014

[14] F. Petitjean, C. Kurtz, N. Passat, P. Ganarski, Spatio-temporal reasoning for the classification of satellite image time series, Pattern Recognition Letters 33 (13) (2012) 1805 - 1815. doi:https://doi.org/10.1016/j.patrec.2012.06.009. URL http://www.sciencedirect.com/science/article/pii/S0167865512001973

[15] F. Guttler, D. Ienco, J. Nin, M. Teisseire, P. Poncelet, A graph-based approach to detect spatiotemporal dynamics in satellite image time series, ISPRS Journal of Photogrammetry and Remote Sensing 130 (2017) 92-107.

[16] B. J. Frey, D. Dueck, Clustering by passing messages between data points, Science 315 (5814) (2007) 972-976. arXiv: http://science.sciencemag.org/content/315/5814/972.full.pdf, doi:10.1126/science.1136800. URL http://science.sciencemag.org/content/315/5814/972

[17] T. Guyet, H. Nicolas, Long term analysis of time series of satellite images, Pattern Recogn. Lett. 70 (C) (2016) 17-23. doi: $10.1016 / j$.patrec. 2015.11 .005 . URL http://dx.doi.org/10.1016/j.patrec.2015.11.005

[18] T. W. Liao, Clustering of time series data survey, Pattern Recognition 38 (11) (2005) 1857 - 1874. doi:https://doi. org $/ 10.1016 / j$.patcog. 2005.01.025. URL http://www.sciencedirect.com/science/article/pii/S0031320305001305

[19] T. Blaschke, ISPRS Journal of Photogrammetry and Remote Sensing Object based image analysis for remote sensing, ISPRS Journal of Photogrammetry and Remote Sensing 65 (1) (2010) 2-16. doi:10.1016/j.isprsjprs.2009.06.004. URL http://dx.doi.org/10.1016/j.isprsjprs.2009.06.004

[20] V. Dey, Y. Zhang, M. Zhong, A review on image segmentation techniques with remote sensing perspective, na, 2010.

[21] Y. Qin, Z. Niu, F. Chen, B. Li, Y. Ban, Object-based land cover change detection for cross-sensor images, International Journal of Remote Sensing 34 (19) (2013) 6723-6737. arXiv:http://dx.doi.org/10.1080/01431161.2013.805282, doi: $10.1080 / 01431161.2013 .805282$. URL http://dx.doi.org/10.1080/01431161.2013.805282

[22] B. Desclée, P. Bogaert, P. Defourny, Forest change detection by statistical object-based method, Remote Sensing of Environment 102 (1) (2006) 1-11.

[23] L. Khiali, D. Ienco, M. Teisseire, Object-oriented satellite image time series analysis using a graph-based representation, Ecological Informatics 43 (2018) 52-64.

[24] A. Huete, A soil-adjusted vegetation index (savi), Remote Sensing of Environment 25 (3) (1988) 295 - 309. doi:https: //doi.org/10.1016/0034-4257(88)90106-X. URL http://www.sciencedirect.com/science/article/pii/003442578890106X

[25] A. R. Huete, H. Liu, G. R. de Lira, K. Batchily, R. Escadafal, A soil color index to adjust for soil and litter noise in vegetation index imagery of arid regions, in: Geoscience and Remote Sensing Symposium, 1994. IGARSS '94. Surface and Atmospheric Remote Sensing: Technologies, Data Analysis and Interpretation., International, Vol. 2, 1994, pp. 1042-1043 vol.2. doi:10.1109/IGARSS.1994.399338.

[26] S. K. McFEETERS, The use of the normalized difference water index (ndwi) in the delineation of open water features, International Journal of Remote Sensing 17 (7) (1996) 1425-1432. arXiv:http://dx.doi.org/10.1080/01431169608948714, doi: $10.1080 / 01431169608948714$. URL http://dx.doi.org/10.1080/01431169608948714

[27] J. R. Jr, R. Haas, J. Schell, D. Deering, Monitoring vegetation systems in the great plains with erts, NASA special 
publication 351 (1974) 309 .

[28] D. Comaniciu, P. Meer, Mean shift: a robust approach toward feature space analysis, IEEE Transactions on Pattern Analysis and Machine Intelligence 24 (5) (2002) 603-619. doi:10.1109/34.1000236.

[29] F. Wan, F. Deng, Remote Sensing Image Segmentation Using Mean Shift Method, Springer Berlin Heidelberg, Berlin, Heidelberg, 2011, pp. 86-90. doi:10.1007/978-3-642-21802-6_14.

URL https://doi.org/10.1007/978-3-642-21802-6\$_\$14

[30] E. Keogh, C. A. Ratanamahatana, Exact indexing of dynamic time warping, Knowledge and information systems 7 (3) (2005) 358-386.

[31] F. Petitjean, J. Inglada, P. Gançarski, Satellite image time series analysis under time warping, IEEE Transactions on Geoscience and Remote Sensing 50 (8) (2012) 3081-3095.

[32] P.-N. Tan, M. Steinbach, V. Kumar, Introduction to Data Mining, (First Edition), Addison-Wesley Longman Publishing Co., Inc., Boston, MA, USA, 2005.

[33] L. Khiali, D. Ienco, M. Teisseire, Object-oriented satellite image time series analysis using a graph-based representation, Ecological Informatics 43 (2018) 52-64.

[34] D. Ienco, R. G. Pensa, R. Meo, From context to distance: Learning dissimilarity for categorical data clustering, ACM Transactions on Knowledge Discovery from Data (TKDD) 6 (1) (2012) 1. 